\title{
Signatures of in-plane and out-of-plane magnetization generated by synchrotron radiation in magnetically doped and pristine topological insulators
}

\author{
A. M. Shikin, ${ }^{1}$ A. A. Rybkina, ${ }^{1}$ D. A. Estyunin, ${ }^{1}$ D. M. Sostina, ${ }^{1}$ V. Yu. Voroshnin, ${ }^{1}$ I. I. Klimovskikh, ${ }^{1}$ A. G. Rybkin, ${ }^{1}$ \\ Yu. A. Surnin, ${ }^{1}$ K. A. Kokh, ${ }^{1,2,3}$ O. E. Tereshchenko, ${ }^{1,2,4}$ L. Petaccia, ${ }^{5}$ G. Di Santo, ${ }^{5}$ P. N. Skirdkov, ${ }^{6,7,8}$ K. A. Zvezdin, ${ }^{6,7,8}$ \\ A. K. Zvezdin, ${ }^{6,7,8}$ A. Kimura, ${ }^{9}$ E. V. Chulkov, ${ }^{1,10,11,12}$ and E. E. Krasovskii ${ }^{10,11,13}$ \\ ${ }^{1}$ Saint Petersburg State University, Saint Petersburg 198504, Russia \\ ${ }^{2}$ Novosibirsk State University, Novosibirsk 630090, Russia \\ ${ }^{3}$ V.S. Sobolev Institute of Geology and Mineralogy, Novosibirsk 630090, Russia \\ ${ }^{4}$ A.V. Rzhanov Institute of Semiconductor Physics, Novosibirsk 630090, Russia \\ ${ }^{5}$ Elettra Sincrotrone Trieste, Strada Statale $14 \mathrm{~km}$ 163.5, 34149 Trieste, Italy \\ ${ }^{6}$ Moscow Institute of Physics and Technology, Institutskiy per. 9, 141700 Dolgoprudny, Russia \\ ${ }^{7}$ A.M. Prokhorov General Physics Institute, Russian Academy of Sciences, Vavilova 38, 119991 Moscow, Russia \\ ${ }^{8}$ Russian Quantum Center, Novaya St. 100, 143025 Skolkovo, Moscow Region, Russia \\ ${ }^{9}$ Graduate School of Science, Hiroshima University, 1-3-1 Kagamiyama, Higashi-Hiroshima 739-8526, Japan \\ ${ }^{10}$ Departamento de Física de Materiales, Facultad de Ciencias Químicas, UPV/EHU, \\ San Sebastián/Donostia, 20080 Basque Country, Spain \\ ${ }^{11}$ Donostia International Physics Center (DIPC), San Sebastián/Donostia, 20018 Basque Country, Spain \\ ${ }^{12}$ Centro de Fisica de Materiales CFM - MPC and Centro Mixto CSIC-UPV/EHU, \\ San Sebastián/Donostia, 20080 Basque Country, Spain \\ ${ }^{13}$ IKERBASQUE, Basque Foundation for Science, 48013 Bilbao, Spain
}

(Received 26 November 2017; published 11 June 2018)

\begin{abstract}
Possibility of in-plane and out-of-plane magnetization generated by synchrotron radiation (SR) in magnetically doped and pristine topological insulators (TIs) is demonstrated and studied by angle-resolved photoemission spectroscopy. We show experimentally and by $a b$ initio calculations how nonequal depopulation of the Dirac cone (DC) states with opposite momenta in V-doped and pristine TIs generated by linearly polarized SR leads to the hole-generated uncompensated spin accumulation followed by the SR-induced magnetization via spintorque effect. Moreover, the photoexcitation of the DC is asymmetric, and it varies with the photon energy. We find a relation between the photoexcitation asymmetry, the generated spin accumulation, and the induced inplane and out-of-plane magnetic field. Experimentally the SR-generated in-plane and out-of-plane magnetization is confirmed by the $k_{\|}$shift of the DC position and by the gap opening at the Dirac point even above the Curie temperature. Theoretical predictions and estimations of the measurable physical quantities substantiate the experimental results.
\end{abstract}

DOI: 10.1103/PhysRevB.97.245407

\section{INTRODUCTION}

The photoexcitation by laser or synchrotron radiation is accompanied by depopulation of the initial states, which influences the electronic structure observed in photoemission (PE) measurements. In materials with helical spin structure like topological insulators (TIs) [1-4] the imbalance in photoexcitation of the DC states with opposite momenta created by circularly polarized laser or SR can be effectively used for generation of the surface spin-polarized currents that depend on the helicity of the radiation polarization [5-10]. Similar to the case of an electric field applied in the surface plane [11-13], this can induce a magnetization in magnetically doped TIs [8,14]. The induced magnetic moment opens a gap at the Dirac point (DP) due to time reversal symmetry (TRS) breaking providing a platform for the realization of quantum phenomena such as quantized magnetoelectric [15-17] and quantum anomalous Hall [18-20] effects at elevated temperatures under optical excitation. Although the possibility of induced (and controlled) magnetization by linearly polarized SR has not been studied yet, the generation of spin-polarized current was noted recently [1,7-9]. The present work aims to investigate a possibility of such SR-induced in-plane and outof-plane magnetization in V-doped and pristine TIs by linearly polarized SR. We relate this phenomenon to an asymmetry in the depopulation of the DC states with opposite momenta, which leads to a hole-generated uncompensated spin accumulation. The possibility of a long-living electron-hole separation between the excited electrons and generated holes (related to a reduced electron-phonon interaction at the surface [21,22]) is confirmed by a series of time-resolved laser experiments (see, for instance, Refs. [21-23]). The holes generated at the topological surface states (TSSs) are compensated by a drift of electrons from the TSSs out of the beam spot via a long-time two-dimensional relaxation process [21]. In the case of a different probability of the photoexcitation of electrons from the DC states with opposite momenta, the uncompensated spin accumulation and the zero-bias surface spin-polarized 
photocurrent occur, as in Refs. [8,9,14], which can lead to induced magnetization similar to the case discussed in Ref. [24] for the (Ga,Mn)As ferromagnetic semiconductor.

The issue of SR-generated spin accumulation is strongly related to the TSS spin texture and to the asymmetry of the photoexcitation of the TSSs with opposite spin orientation. In Refs. [25,26], it was shown that a total spin texture of TSSs includes not only contributions of the $p_{z}$ orbitals, but also of the $p_{x}$ and $p_{y}$ orbitals related to the radial and tangential components in the spin-orbital texture, which significantly modifies the spin texture of the TSSs probed by photoemission. Total spin structure in PE spectra depends on the sum of all contributions determined by optical selection rules, quantum interference, and SR incident angle [27-29]. Due to variation of $k_{z}$ with photon energy all $p_{x, y, z}$ components "oscillate" with different phases. The nontrivial character of TSS spin texture is confirmed by spin-resolved photoemission both via theoretical analysis [26-32] and via experimental measurements [26-29] using different polarization of SR [27,28,30,33] and manifests itself in significant modification of the spin polarization of photoelectrons with photon energy [27,28,30,31]. The oblique incidence of SR breaks the symmetry of the angular distribution of the photocurrent, and the different photoemission intensity at $k_{\|}$and $-k_{\|}[26,27]$ can be a source of the SR-generated uncompensated spin accumulation and an induced magnetization. This problem is especially important for magnetically doped TIs, because the DP gap opening due to the TRS breaking, its value and origin (magnetization or hybridization derived) are being actively discussed; see, for instance, Ref. [34]. However, without a proper analysis of the influence of the nonequal depopulation of the TSSs on a possible induced magnetization during angle-resolved photoemission spectroscopy (ARPES) and spin-resolved ARPES (SARPES) measurements these questions cannot be answered.

In the present work we study how the photoemission intensity asymmetry of the DC states and the connected SRgenerated asymmetric $k_{\|}$distribution of the holes can induce a local in-plane and out-of-plane surface magnetization via the generated uncompensated spin accumulation due to spintorque effect. The first part of the work aims to clarify how the photoexcitation by linearly polarized SR influences the DC states intensity distribution in the ARPES intensity maps and how it varies with photon energy. In the second part we analyze how the imbalance in the depopulation of the TSSs in V-doped TI and the related hole-generated uncompensated spin accumulation result in inducing the in-plane and out-of-plane surface magnetization of TSSs and the V $3 d$ ions.

At the same time, we have to note that the magnetically doped TIs are typically characterized by the surface Curie temperature significantly higher than the bulk one [35]. Therefore, they can be considered in certain temperature ranges as $2 \mathrm{D}$ material with surface magnetic layer on top of paramagnetic bulk, which is characterized by enhanced spin-orbit coupling (SOC). Due to the same crystalline parameters for magnetic surface and paramagnetic bulk, these can now be considered as very promising 2D magnetic systems allowing one to realize the so called magnetic "extension" between the 2D magnetic layer and the bulk of TI (allowing the direct interaction of the DC states with magnetic layer) instead of the magnetic proximity effect observed for ferromagnetic layer on top of TI [36,37].
In the current work we analyze how the local surface in-plane and out-of-plane magnetic field can be developed in such kind of 2D magnetic materials under nonequal photoexcitation of the DC states with opposite momenta at different photon energy.

\section{ASYMMETRY IN THE INTENSITY OF THE DC STATES VS PHOTON ENERGY}

Here we analyze both experimentally and theoretically how the oblique incidence of SR on TI samples is followed by the asymmetry in photoexcitation of the TSSs with opposite momentum estimating the photon-energy dependence of the generated PE TSS intensity asymmetry. In the current work we study a series of the $\mathrm{V}$-doped and pristine TIs with fractional stoichiometry based on $\mathrm{Bi}_{2} \mathrm{Te}_{2} \mathrm{Se}$ with inclusion of different $\mathrm{Sb}$ concentrations. These TIs have a wide insulating energy gap with the DP inside the bulk gap [38,39] and are characterized by an enhanced surface contribution to the spin transport [40], which is important for spintronic applications.

Figures 1(a)-1(c) show the photon energy dependence of the TSS ARPES energy-momentum intensity maps measured along the $\overline{\Gamma K}$ direction of the surface Brillouin zone using linearly $p$-polarized SR for pristine and magnetically doped TIs with the stoichiometries, (a) $\mathrm{Bi}_{1.5} \mathrm{Sb}_{0.5} \mathrm{Te}_{1.8} \mathrm{Se}_{1.2}$, (b) $\mathrm{Bi}_{2} \mathrm{Te}_{2} \mathrm{Se}$, and (c) $\mathrm{Bi}_{1.37} \mathrm{~V}_{0.03} \mathrm{Sb}_{0.6} \mathrm{Te}_{2} \mathrm{Se}$, when the dispersions were measured along the SR incidence plane. The presented ARPES dispersion maps were measured at temperature 17-20 $\mathrm{K}$. One can see a pronounced asymmetry in photoexcitation of the opposite branches of the DC states characterized by opposite momentum. Similar ARPES dispersions measured perpendicular to the $\mathrm{SR}$ incidence plane in the direction along $\overline{\Gamma M}$ for $\mathrm{Bi}_{1.5} \mathrm{Sb}_{0.5} \mathrm{Te}_{1.8} \mathrm{Se}_{1.2}$ are shown in the Supplemental Material [41] (Fig. 1S), for comparison. For the last geometry, which is often used for ARPES experiments, the asymmetry in the TSS intensity is less pronounced. The geometries of the experiments are schematically shown in the Methods section, Fig. 11, and are marked as Geometries $\mathbf{1}$ and $\mathbf{2}$. They differ by the analyzer entrance slit orientation along and perpendicular to the SR incidence plane, respectively. For Geometry 1 two SR incidence angles $(\theta)$ have been used: $73^{\circ}$, Fig. 1(a), and $50^{\circ}$, Figs. 1(b), 1(c) relative to the surface normal. For Geometry 2 the incidence angle was $45^{\circ}$. The intensity maps for $\mathrm{Bi}_{1.5} \mathrm{Sb}_{0.5} \mathrm{Te}_{1.8} \mathrm{Se}_{1.2}$ measured at room temperature along $\overline{\Gamma M}$ (Geometry 1), for $\mathrm{Bi}_{1.47} \mathrm{~V}_{0.03} \mathrm{Sb}_{0.5} \mathrm{Te}_{2}$ Se along $\overline{\Gamma K}$ (Geometry 1) and for $\mathrm{Bi}_{1.5} \mathrm{Sb}_{0.5} \mathrm{Te}_{1.8} \mathrm{Se}_{1.2}$ along $\overline{\Gamma M}$ (Geometry 2), are presented in the Supplemental Material [41] (Fig. 2S) for comparison. Below each ARPES intensity map the profiles of the TSS intensities, as momentum distribution curves (MDCs), are presented for the constant-energy cut of the upper DC at the binding energy corresponding to a high intensity of the TSSs (white lines in the ARPES maps). In all the profiles the intensities at opposite $k_{\|}$points are different. At some photon energies the intensity at positive $k_{\|}$is larger than at negative $k_{\|}$, and at other photon energies the relation is opposite. Figure 2 and Fig. 1S collect all data measured for different TIs and demonstrate the photon energy dependence of the asymmetry in the intensity of the opposite TSS branches, more pronounced for Geometry 1 . The TSS intensity asymmetries are defined by 


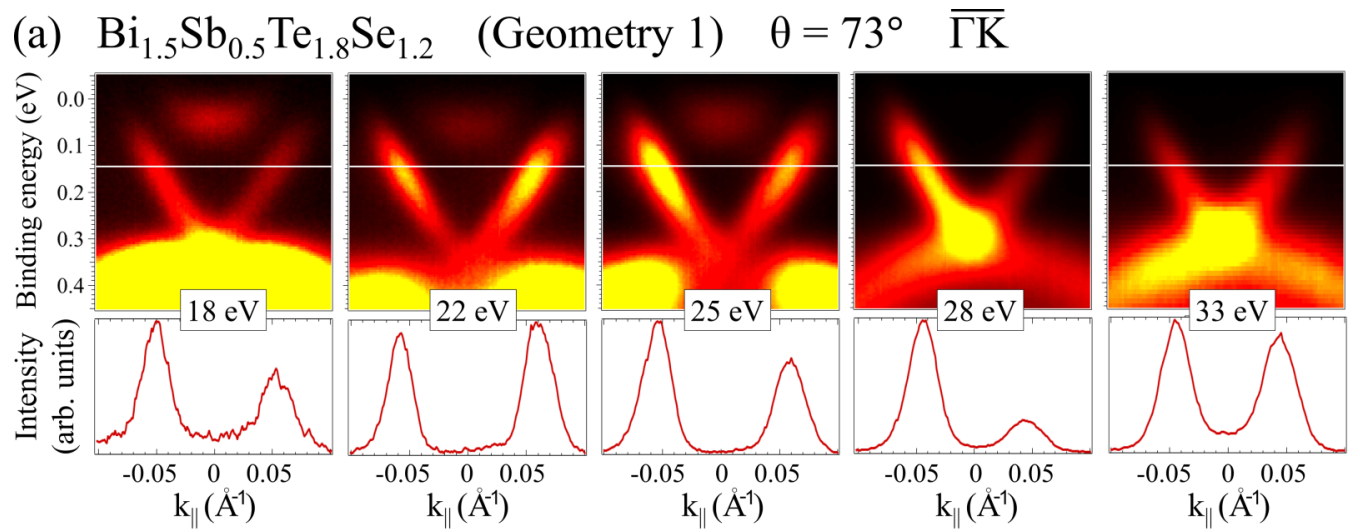

(b) $\mathrm{Bi}_{2} \mathrm{Te}_{2} \mathrm{Se}$ (Geometry 1) $\quad \theta=50^{\circ} \quad \overline{\Gamma \mathrm{K}}$

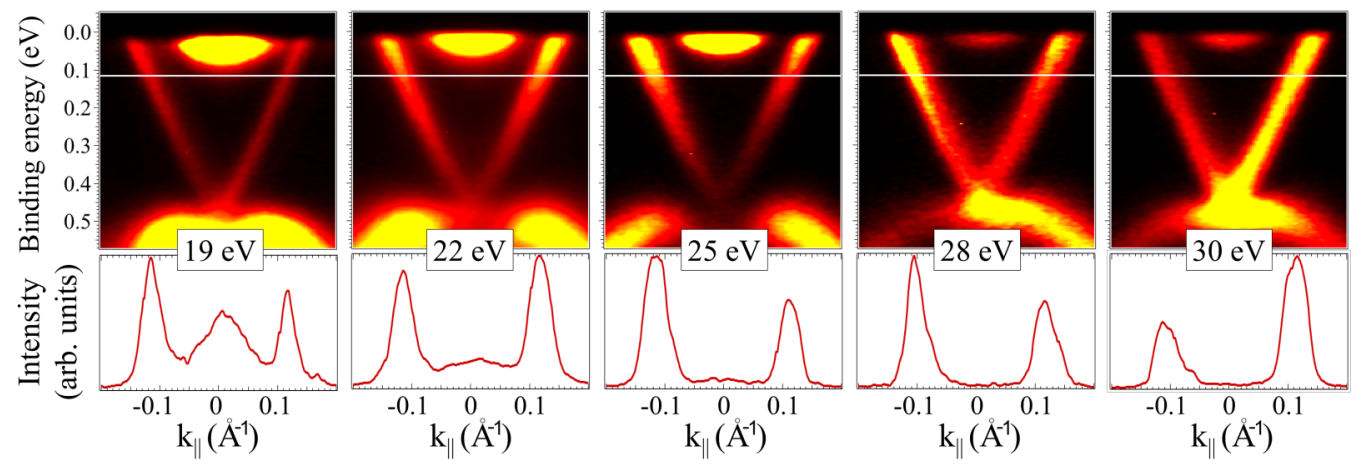

(c) $\mathrm{Bi}_{1.37} \mathrm{~V}_{0.03} \mathrm{Sb}_{0.6} \mathrm{Te}_{2} \mathrm{Se} \quad$ (Geometry 1) $\quad \theta=50^{\circ} \quad \overline{\Gamma \mathrm{K}}$
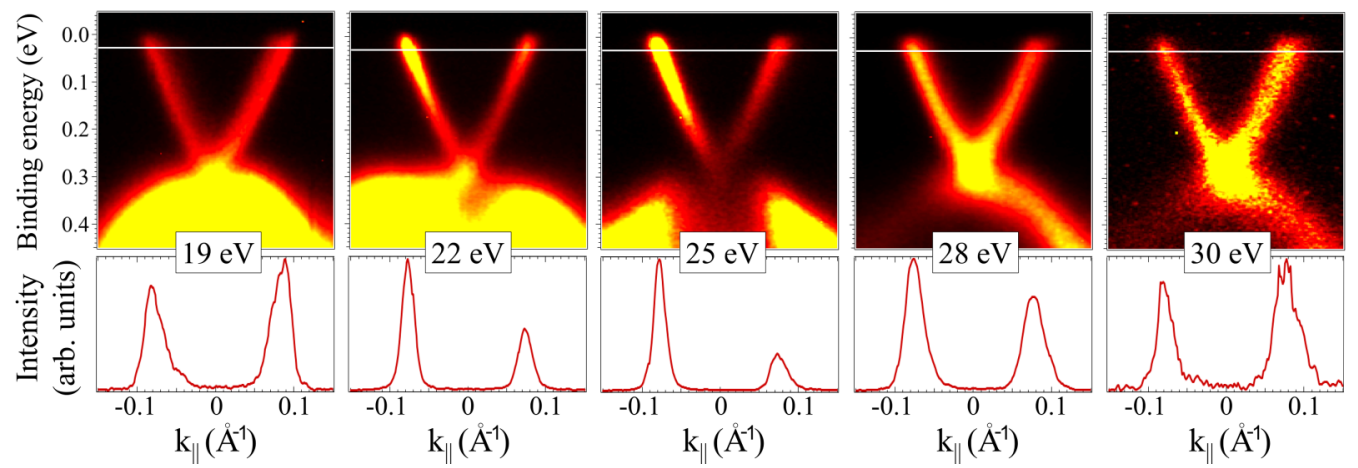

FIG. 1. Lines (a),(b),(c): series of ARPES intensity maps of the TSSs measured along the SR incidence plane $(\overline{\Gamma K})(\mathrm{Geometry} 1)$ for pristine TIs with stoichiometry $\mathrm{Bi}_{1.5} \mathrm{Sb}_{0.5} \mathrm{Te}_{1.8} \mathrm{Se}_{1.2}$ (a) and $\mathrm{Bi}_{2} \mathrm{Te}_{2} \mathrm{Se}$ (b) and for V-doped TIs with stoichiometry $\mathrm{Bi}_{1.37} \mathrm{~V}_{0.03} \mathrm{Sb}_{0.6} \mathrm{Te}_{2} \mathrm{Se}(\mathrm{c})$ at temperature 17-20 K by using linear $p$-polarized SR at different photon energy. The profiles of comparable intensities of the TSSs with opposite momenta (MDC) cut at the energy corresponding to enhanced intensity (marked by horizontal white lines) are presented at the bottom of each inset.

the ratio

$$
A=\frac{I\left(-k_{\|}\right)-I\left(k_{\|}\right)}{I\left(-k_{\|}\right)+I\left(k_{\|}\right)},
$$

measured at the energies marked by white horizontal lines. [For Fig. 2 and Fig. 1S(b) we used the height of the peaks on the MDC profiles to avoid a possible dependence of the peaks structure.] One can see that the asymmetry "oscillates" with photon energy both for pristine and for V-doped TIs. As we will show later, this TSS intensity asymmetry is necessary for the generation of the uncompensated spin accumulation accompanied by the SR-generated surface magnetization.
In order to theoretically confirm and explain the observed $k_{\|}$distribution of the photocurrent and its variation with the photon energy we have performed ab initio calculations of the energy-momentum distribution of the photoemission intensity from the DC of the stoichiometric compound $\mathrm{Bi}_{2} \mathrm{Te}_{2} \mathrm{Se}$. The electronic structure of $\mathrm{Bi}_{2} \mathrm{Te}_{2} \mathrm{Se}$ [38] is quite similar to that of the crystals with the fractional stoichiometry studied here. We use the one-step theory of photoemission in the dipole approximation, so the photocurrent from the state $\left|\mathbf{k}_{\|}\right\rangle$is proportional through the transition probability $\left|\left\langle\Phi\left|-i \nabla_{\mathbf{e}}\right| \mathbf{k}_{\|}\right\rangle\right|^{2}$ to the time-reversed low energy electron diffraction state $|\Phi\rangle$ [42], where $-i \nabla_{\mathbf{e}}$ is the momentum operator in the direction of 

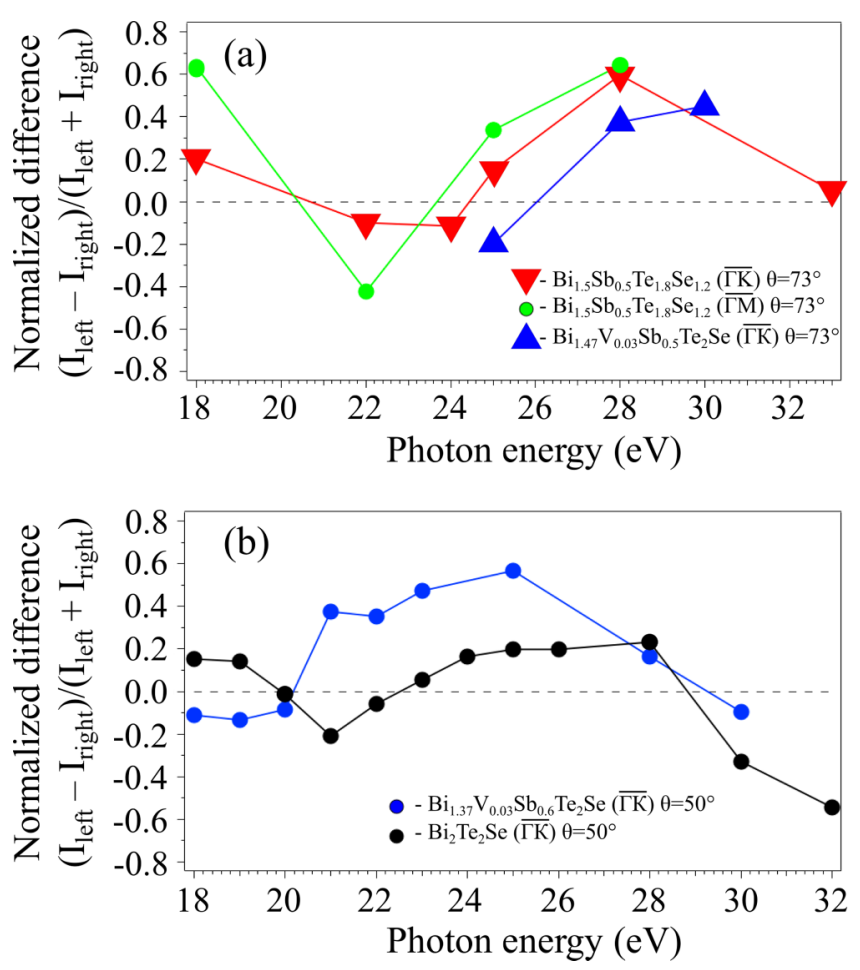

FIG. 2. TSS intensity asymmetry ( $A$ ) trend vs photon energy estimated from the ARPES intensity maps presented in Figs. 1(a)-1(c) and Figs. 1S, 2S in the Supplemental Material [41] measured along $\overline{\Gamma K}$ and $\overline{\Gamma M}$ directions of the surface Brillouin zone.

the light polarization e. The final state $|\Phi\rangle$ is calculated for the scattering of electrons on a semi-infinite crystal as explained in Ref. [43]. The inelastic scattering is included by adding a spatially constant imaginary part $V_{\mathrm{i}}=1 \mathrm{eV}$ to the crystal potential. The crystal potential was obtained within the local density approximation with the full-potential linear augmented plane wave method [44]. The initial states were calculated within a two-component relativistic formalism [45] for a slab composed of seven quintuple layers. Figures 3(a)-3(c) show the $k_{\|}$distribution of the calculated photoemission intensity from the $\mathrm{DC}$ of $\mathrm{Bi}_{2} \mathrm{Te}_{2} \mathrm{Se}$ along $\overline{\Gamma K}$ direction (Geometry 1) and Figs. 3(d)-3(f) along $\overline{\Gamma M}$ direction (Geometry 2). In both cases the $p$-polarized SR is incident along $\overline{\Gamma K}$ direction. The $k_{\|}$-integrated intensities for opposite directions of $k_{\|}$are shown in Figs. 3(g) and 3(h), respectively. The corresponding asymmetry index for the light incident along $k_{\|}$(Geometry 1) is shown in Fig. 3(i) and for the light incident perpendicular to $k_{\|}$(Geometry 2) in Fig. 3(j).

Note that when the light propagates along $k_{\|}$the difference between $+k_{\|}$and $-k_{\|}$is due to a linear dichroism, and it strongly depends on the angle of incidence; see Fig. 3(i). In particular, along $\overline{\Gamma K}$ the opposite directions are equivalent owing to the $C_{3 v}$ symmetry of the surface, and it is the light that breaks the symmetry of the experiment. On the contrary, for the light propagating perpendicular to $\mathbf{k}_{\|}$the intensity asymmetry is due to the inequivalence of $+k_{\|}$and $-k_{\|}$along $\overline{\Gamma M}$. In that case the photon energy dependences $I_{\text {left }}(h \nu)$ and $I_{\text {right }}(h v)$ are very similar, and the intensity asymmetry does not strongly depend on $\theta$; see Fig. 3(j). The theory explains the experimentally observed oscillations of the asymmetry index with the photon energy and relates them to energy variations of the final state $|\Phi\rangle$.

Therefore, the intensity asymmetry of the TSSs with opposite momentum and its variation with the photon energy are well predicted by our theory, which corroborates the experimental ARPES results. In the following, we analyze the relation between the observed TSS asymmetry and the SR-induced magnetic field.

\section{HOLE GENERATION ANALYSIS AND ASYMMETRY VARIATION DURING RELAXATION}

Let us assume that at the used SR energies the excited spin-polarized photoelectrons mainly escape into the vacuum. Then, the uncompensated spin accumulation generated by the different photoexcitation rate of electrons with opposite momentum is mainly determined by the asymmetry in the concentration of corresponding photoholes in the opposite TSS branches characterized by opposite momentum and spin orientation. In the simplest photoemission approximation one can infer the photohole concentration asymmetry and the resulting uncompensated spin accumulation from the experimentally observed asymmetry of intensity of the DC states with opposite momenta. We assume that the TSS intensity asymmetry generated under photoexcitation is not strongly changed within the relaxation time. Some qualitative arguments for it are presented in the Supplemental Material [41] (Figs. 4S and 5S) and in Ref. [46]. In other words the photohole generated asymmetry does not strongly change with the relaxation time, and the asymmetry in the measured PE intensity of the TSSs with opposite momenta can be used for a rough estimate of the induced uncompensated spin accumulation and the related surface magnetization.

In the following we discuss the theoretical estimations of the magnetization induced by linear $p$-polarized SR due to the generated uncompensated spin accumulation. First of all, let us consider the asymmetry of the spin accumulation induced by linearly polarized SR. The speed of spin density generation $(\dot{\vec{\Sigma}})$ due to electromagnetic radiation is the time-even pseudovector. Therefore, its components, according to the crystal symmetry (see, e.g., Refs. [47,48]), in the second order on electric field components can be present in the form [49]

$$
\Sigma_{i}=B_{i k l} E_{k} E_{l},
$$

where $B_{i k l}$ is the third rank pseudotensor and $E_{k}$ are the components of electric field. Following Refs. [50,51] the studied Bi-based compounds have the space group $D_{3 d}^{5}(R \overline{3} m)$. In the presence of a [111] surface, the symmetry of the considered four-component complex compound can be reduced to $C_{3}$ and, therefore, $B_{i k l}$ is equal [49]:

$$
B_{i k l}=\left(\begin{array}{cccccc}
B_{11} & -B_{11} & 0 & B_{14} & B_{15} & -B_{22} \\
-B_{22} & B_{22} & 0 & B_{15} & -B_{14} & -B_{11} \\
B_{31} & B_{31} & B_{33} & 0 & 0 & 0
\end{array}\right),
$$

where $B_{i \mu}=B_{i k l}(k l \leftrightarrow \mu=1, \ldots, 6)$ are the coefficients determined by the material. Considering the SR as $\vec{E}=$ ( $E \cos \psi, 0, E \sin \psi)^{T}$, from Eq. (3) it immediately follows 


\section{Geometry 1}
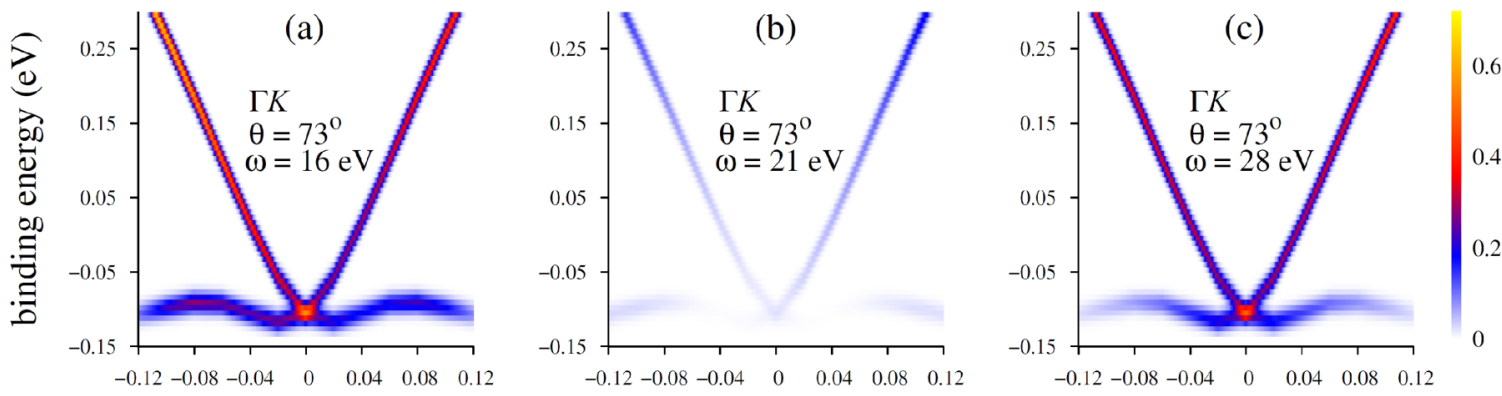

Geometry 2
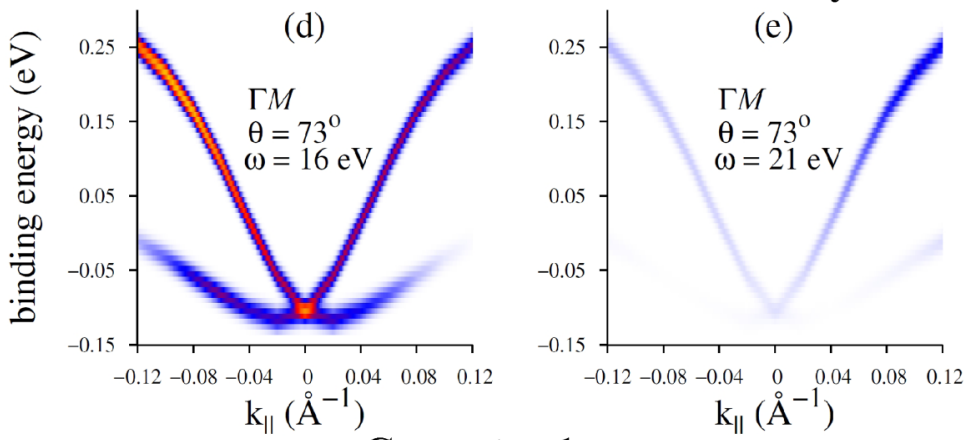

Geometry 1
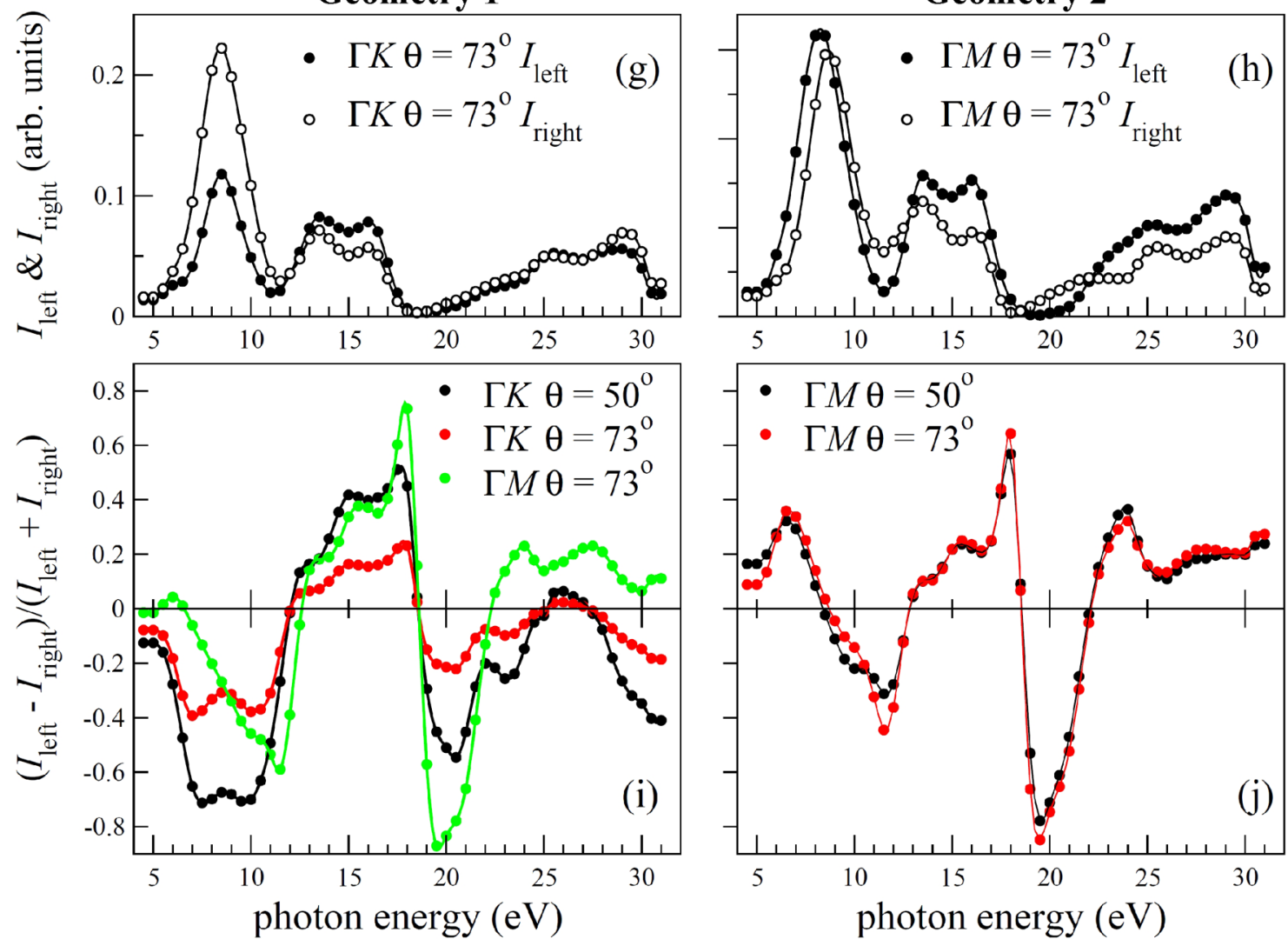

FIG. 3. (a)-(f) Calculated energy-momentum distribution of the photoemission intensity from the DC surface states along $\bar{\Gamma} \bar{K}$ (Geometry 1 ) (a)-(c) and along $\bar{\Gamma} \bar{M}$ (Geometry 2) (d)-(f) for the three photon energies: $h \omega=16$ (a),(d), 21 (b),(e), and $28 \mathrm{eV}$ (c),(f). In all cases a $p$-polarized light is incident along $\bar{\Gamma} \bar{K}$ at an angle of $\theta=73^{\circ}$. (g),(h) Photon energy dependence of the total intensity from the upper Dirac cone integrated over negative $k_{\|}\left(I_{\text {left }}\right.$, full circles) and positive $k_{\|}\left(I_{\text {right }}\right.$, open circles) along $\bar{\Gamma} \bar{K}(\mathrm{~g})$ and along $\bar{\Gamma} \bar{M}(\mathrm{~h})$. (i), (j) Normalized difference between the integrated intensities in the opposite directions, $\left[I_{\text {left }}(h v)-I_{\text {right }}(h v)\right] /\left[I_{\text {left }}(h v)+I_{\text {right }}(h v)\right]$, for two angles of incidence, $\theta=50^{\circ}$ and $73^{\circ}$. In graph (i) the light incidence plane is parallel to $\mathbf{k}_{\|}$(both for $\bar{\Gamma} \bar{K}$ and for $\bar{\Gamma} \bar{M}$ ), and in graph (j) it is perpendicular to $\mathbf{k}_{\|}(\bar{\Gamma} \bar{M})$, i.e., Geometry 1 and 2, respectively. 


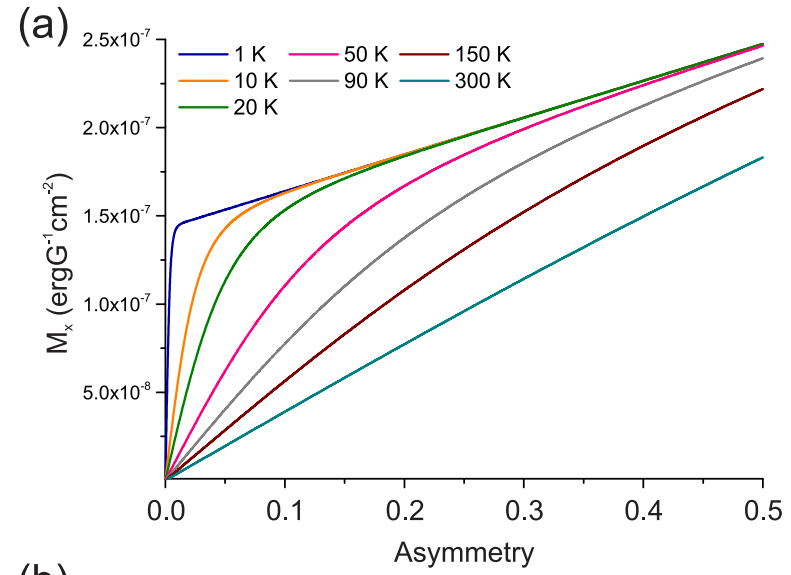

(b)

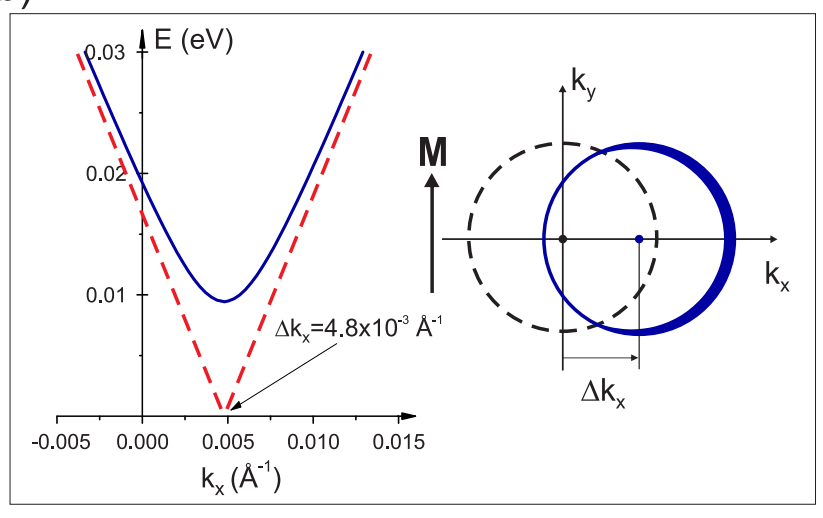

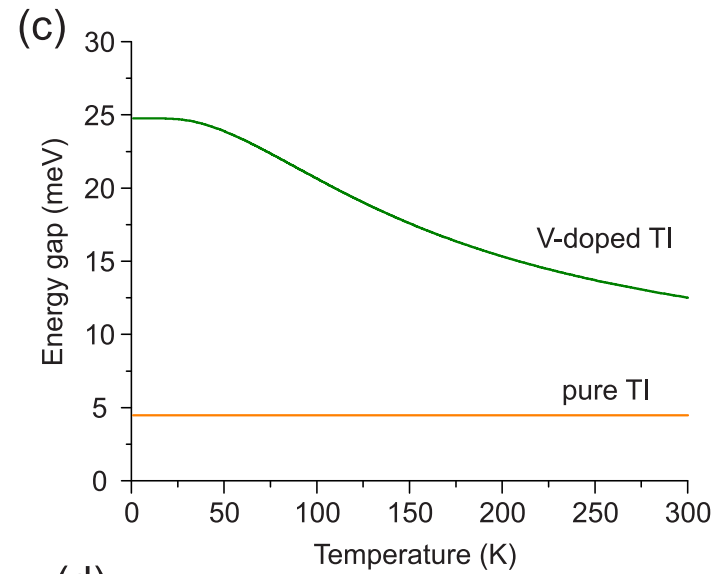

(d)

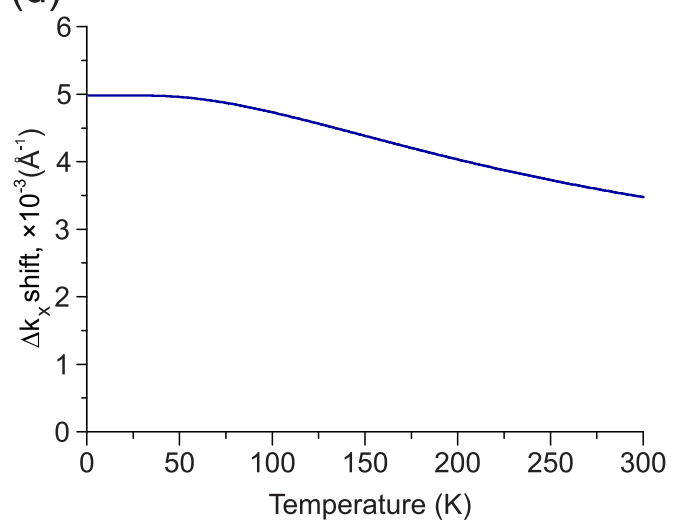

FIG. 4. (a) Calculated dependence of the in-plane component of a total SR-induced magnetization vs the asymmetry in the hole generation with opposite spins (A) for different temperatures between 1 and $300 \mathrm{~K}$. (b) Modification of the DC state dispersions obtained by the plane crossing along $k_{x}$ (at $k_{y}=0$ ) which indicate the influence of the out-of-plane and in-plane components of induced magnetization, i.e., opening the gap at the DP and the $k_{x}$ shift of the DC states, respectively. The solid lines represent the modified upper DC state dispersion, while the dotted ones show the $k_{\| \mid}$shift of the DC without the gap opening. (c),(d) Calculated temperature dependences of the energy gap at the DP due to the out-of-plane component of the induced magnetization for magnetically doped and pristine TIs and corresponding $k_{\|}$shift of the DP relative to $k_{\|}=0$ in the direction orthogonal to the magnetization as a function of temperature.

that SR can generate both the in-plane and out-of-plane uncompensated spin accumulation. Assuming that the linearly polarized SR can be decomposed into right and left circularly polarized SR and each of them can depopulate mostly one of the Dirac cone branches [14], one can write the spin-density derivative as the sum of photoexcitation speed $P$ and relaxation of the spin density $-\Sigma_{i} / \tau$. Hence the stationary averaged spin density (spin accumulation) can be represented as $\delta S_{x, z}=$ $\frac{\hbar}{2} \xi_{x, z} P \tau A$, where $P$ is the probability of the hole generation related to corresponding electron photoexcitation per unit time, $\tau$ is the decoherence time of the hole-generated spin, $\xi_{x, z}$ is an empirical constant, and $A$ is the TSS photoexcitation asymmetry. It should be noted that $P \tau$ is the steady-state concentration of the generated holes. In the simplest case of semiconductor optical orientation $\xi_{x}^{0}=\sin \psi$ and $\xi_{z}^{0}=$ $\cos \psi$ [52]. For simplicity, we assume that $\xi_{x, z}=\kappa_{x, z} \xi_{x, z}^{0}$, where $\kappa_{x, z} \approx 1$, and that the magnetization of an electron subsystem induced by linearly polarized SR can be estimated as $m_{x, z}=\mu_{B} \kappa_{x, z} \xi_{x, z}^{0} P \tau A$, where $\mu_{B}$ is the Bohr magneton (see also [14]).

In the case of the $\mathrm{V}$-impurity subsystem, below the Curie temperature $\left(T<T_{C}\right)$ the total energy can be represented as $E_{V}=-K_{U}\left(m_{z}^{V}\right)^{2}-\left(\vec{m}^{V} \cdot \vec{H}_{S R}\right)$, where $K_{U}$ is the constant of uniaxial anisotropy, $m_{z}^{V}$ is the $z$-axis component of the Vsubsystem magnetization, $\vec{H}_{S R}=H_{S R}\left(\kappa_{x} \sin \psi, 0, \kappa_{z} \cos \psi\right)^{T}$ is the field acting on the $\mathrm{V}$ impurities from the SR induced magnetization, $H_{S R}=\frac{\tilde{a}^{2}}{\mu_{B}} J_{e V} P \tau A$, where $\tilde{a}=4.24 \dot{A}$ is the lattice constant typical of the studied TIs, and $J_{e V} \approx 0.3 \mathrm{eV}$ $[35,53,54]$ is the exchange constant, which describes the $(s-d)$ interaction between the TSSs and the V-ion impurities system. For $\left(T>T_{C}\right)$, the energy minimization leads to the following expression for the $\mathrm{V}$-subsystem magnetization:

$$
\vec{m}^{V}=g \mu_{B} S N B_{S}\left(g \mu_{B} S H_{S R} / T\right)(\sin \eta, 0, \cos \eta)^{T},
$$

where $g \approx 2$ is the $g$ factor, $S=3 / 2$ is the spin of impurity ion, $N$ is the averaged impurity concentration, $B_{S}$ is the Brillouin function, and $\eta$ slightly differs from $\psi$ and $\sin \eta=$ $\kappa_{x} \sin \psi / \sqrt{\kappa_{x}^{2} \sin ^{2} \psi+\kappa_{z}^{2} \cos ^{2} \psi}$. For $\left(T<T_{C}\right)$, we also have to consider anisotropy, but our estimations prove that the anisotropy term is significantly smaller than the SR term (for details, see the Supplemental Material [41]); therefore, Eq. (4) is valid with a good accuracy for all temperature range. The estimated dependence of the in-plane component of the total magnetization $\vec{M}=\vec{m}+\vec{m}^{V}$ on the in-plane asymmetry value (A) for different temperatures is represented in Fig. 4(a). 
For the estimations we have used the experimental parameters such as the SR incidence angle $\left(\psi=50^{\circ}\right)$, photon flux $\left(5(\times) 10^{12}\right.$ photon $\left./ \mathrm{s}\right)$, the SR spot size $(50 \mu \mathrm{m})$, concentration of magnetic impurities, and others noted above. However, the decoherence time of the hole-generated spins and an influence on the holes generated in the subsurface region (which can also participate in inducing magnetic field) remain unknown now. As a first approximation we used the decoherence time value of $50 \mu$ s (in accordance to Ref. [21]) and introduced an empirical constant to reach a correlation with the experimental data. It allowed us to present the expected modification of the DC state dispersion (see Fig. 4) induced by the SR-generated in-plane and out-of-plane magnetization in dependence on the TSS asymmetry and temperature and confirmed the validity of the other used parameters noted before.

The complete electron Hamiltonian of the considered system including both electron-electron and electron-vanadium interactions in the mean field approximation can be written as

$$
\hat{H}_{e}=\hbar V_{D}[\vec{k} \times \vec{\sigma}] \vec{e}_{z}+\frac{\tilde{a}^{2}}{\mu_{B}} J_{e V}\left(\vec{m}^{V} \cdot \vec{\sigma}\right)+\frac{\tilde{a}^{2}}{\mu_{B}} U(\vec{m} \cdot \vec{\sigma}),
$$

where $V_{D} \sim 5.3 \times 10^{7} \mathrm{~cm} / \mathrm{s}$ is the velocity taken from the TSS dispersion law, $\vec{\sigma}$ is the vector of Pauli matrices, and $U \approx$ $0.2 \mathrm{eV}$ is the Hubbard parameter. The energy spectrum in this case has the following form:

$$
E= \pm \sqrt{\hbar^{2} V_{D}^{2}|\vec{k}|^{2}+\Delta_{x}^{2}+\Delta_{z}^{2}-2 \hbar V_{D} k_{y} \Delta_{x}}
$$

where

$$
\begin{aligned}
& \Delta_{x}=\frac{\tilde{a}^{2}}{\mu_{B}} J_{e V} m_{x}^{V}+\frac{\tilde{a}^{2}}{\mu_{B}} U m_{x}, \\
& \Delta_{z}=\frac{\tilde{a}^{2}}{\mu_{B}} J_{e V} m_{z}^{V}+\frac{\tilde{a}^{2}}{\mu_{B}} U m_{z} .
\end{aligned}
$$

The resulting calculated TSS structure modification for $\mathrm{V}$-doped TI with 3\% of V doping under influence of the induced out-of-plane and in-plane magnetization is shown in Fig. 4(b) (under experimental conditions noted above and the value of the asymmetry of $A=0.3$ at photon energy of $28 \mathrm{eV}$ averaged from Fig. 2 for qualitative comparison with the experimental results and analysis of experimental tendencies). In this case the $k_{\|}$shift of about $5 \times 10^{-3} \AA^{-1}$ is expected relative to the $\bar{\Gamma}$ point (if we compare the $k_{\|}$shift between the cases of opposite circular polarizations then it should be twice as large). The out-of-plane magnetization is accompanied by the splitting of the DC states at the DP (i.e., band gap opening). The induced in-plane magnetization manifests itself in the $k_{\|}$shift of the DC in the direction orthogonal to the induced magnetic field (or magnetization) related to the photoemission TSS intensity asymmetry. A similar $k_{\|}$shift is observed under an external applied in-plane magnetic field $[8,55,56]$. The relevant calculated temperature dependence of the DP-gap value and the $k_{\|}$shift of the DP position in the direction orthogonal to the magnetization are presented in Figs. 4(c) and 4(d) (see the discussion below).

\section{Experimental confirmation of the induced in-plane magnetization}

As an experimental test of the SR-induced in-plane magnetization, which can be indicated by the $k_{\|}$shift of the DC, Fig. 5 demonstrates a correlation between the inversion of the TSS intensity asymmetry in the experimental intensity maps measured close to the Fermi level [Fig. 5(a)] and the $\left(k_{x}, k_{y}\right)$ shift of the DP position generated by SR with linear $p$ and opposite circular polarizations [Fig. 5(b)], which is expected in accordance with the corresponding induced in-plane magnetization; see Fig. 4(b). The incident direction of SR corresponds to the vertical line $\left(k_{y}\right)$ in the presented maps. The maps were measured with $h v=28 \mathrm{eV}$ for $\mathrm{Bi}_{1.37} \mathrm{~V}_{0.03} \mathrm{Sb}_{0.6} \mathrm{Te}_{2} \mathrm{Se}$ kept at the temperature of $55 \mathrm{~K}$. The DP positions [Fig. 5(b)] were estimated from the maximal intensity of the TSS in the MDC profiles in $k_{x}$ and $k_{y}$, shown at the bottom and on the right side of the maps at the Dirac energy. Here the position of $k_{\|}=0$ corresponds to that of the maximum in the MDC profile measured at the DP using linearly polarized SR. The MDC profiles measured at the DP and close to the Fermi level in the $k_{x}$ direction under photoexcitation by circularly polarized SR are shown in detail in Fig. 5(c), for comparison. The different depopulation of the opposite DC branches under photoexcitation by SR of opposite chirality [Fig. 5(a)] leads to the $\left(k_{x}, k_{y}\right)$ shift of the DP according to the direction of induced magnetic field, which is determined by the asymmetry in the TSS intensity. The direction of the uncompensated spin accumulation and of the induced in-plane magnetic field are determined by the direction of the TSS intensity asymmetry orientation [along $k_{x}$ in Fig. 5(a)] and by the details of the spin texture [25-27]. The relation between the TSS intensity asymmetry $(A)$, the direction of the induced magnetic field $(M)$ generated by uncompensated spin accumulation, and direction of the DP position $\left(k_{x}, k_{y}\right)$ shift (in the model of the rigid shift of the DC states) is schematically shown in Fig. 4(b). For circularly polarized SR a pronounced asymmetry in the TSS intensity is mainly observed in the direction perpendicular to the SR incidence. The use of the opposite circular polarizations leads to the TSS intensity asymmetry and generated uncompensated spin accumulation with spin orientation (and corresponding magnetic moment) in opposite $k_{\|}$directions. The direction of the induced magnetic field determines the shift of the DP orthogonal to the SR incidence $\left(k_{x}\right)$ that is confirmed experimentally [see the opposite shift of blue crosses measured at opposite circular polarization of SR in comparison with the green one measured at linear polarization of SR in Fig. 5(b)]. The measurements at room temperature (see Fig. 6S in Supplemental Material [41]) and at $30 \mathrm{~K}$ (not shown) at the 9B beamline HiSOR (Hiroshima, Japan) albeit with a lower intensity of SR demonstrate similar behavior, even if the $k_{\|}$-shift value was less due to lower SR intensity.

The $k_{\|}$shift of the DC states induced by linearly polarized SR shown in Fig. 5 (in comparison with the DC position under excitation by circularly polarized SR in the $k_{y}$ direction) is estimated to be approximately $(5-6) \times 10^{-3} \AA^{-1}$ that is in agreement with the theoretical estimate of the $k_{\|}$shift in Fig. 4(b). The lower value of the $k_{\|}$shift of the DP position at room temperature presented in the Supplemental Material [41] 
(a) Cuts at the Fermi level $(\approx 0.02 \mathrm{eV} B E)$
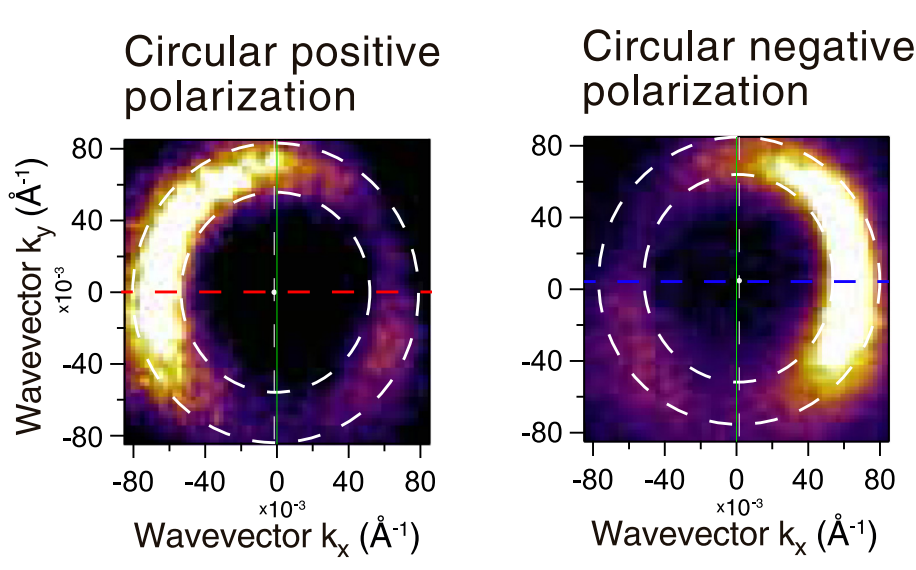

(b) Cuts at the Dirac point $(\approx 0.19 \mathrm{eV} B E)$ (c)
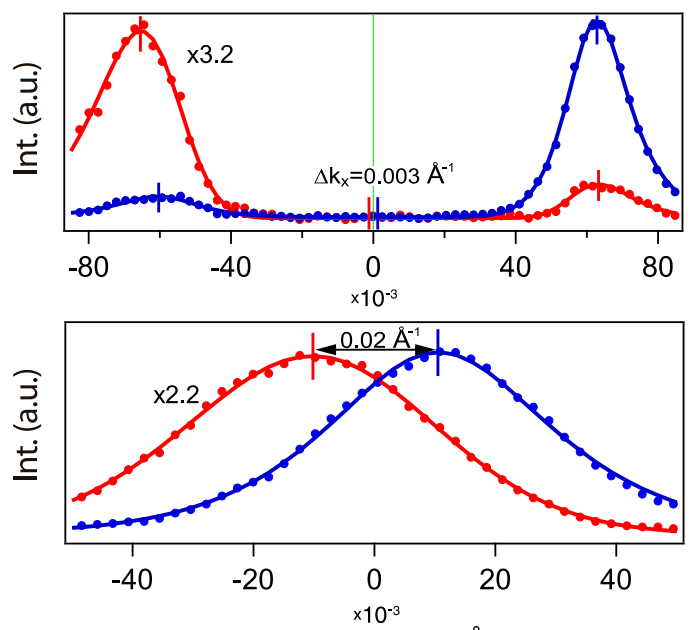

Wavevector $k_{x}\left(\AA^{-1}\right)$

\section{Linear p-polarization}

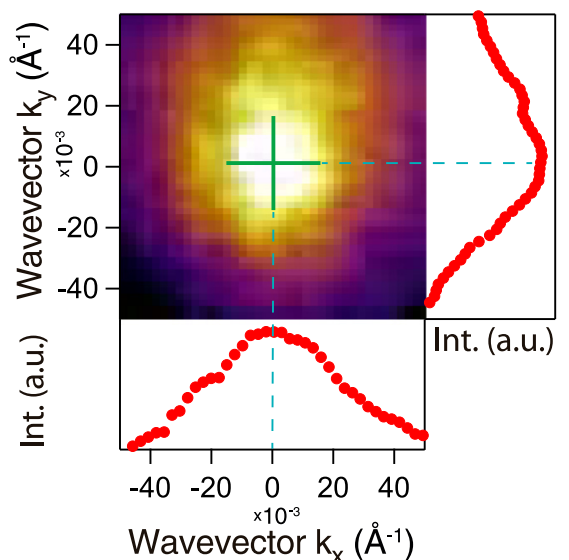

\section{Circular positive polarization}

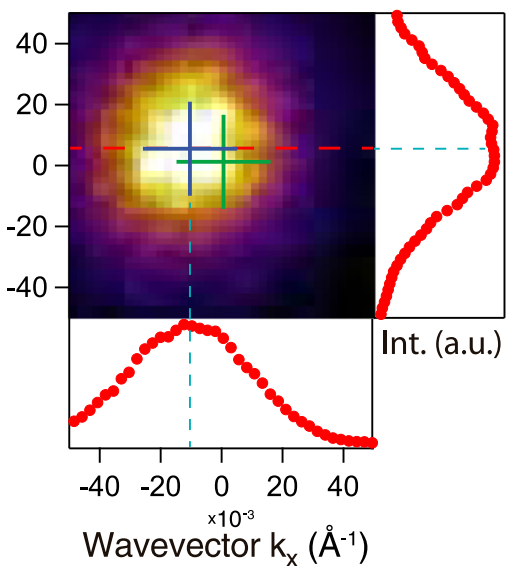

\section{Circular negative polarization}

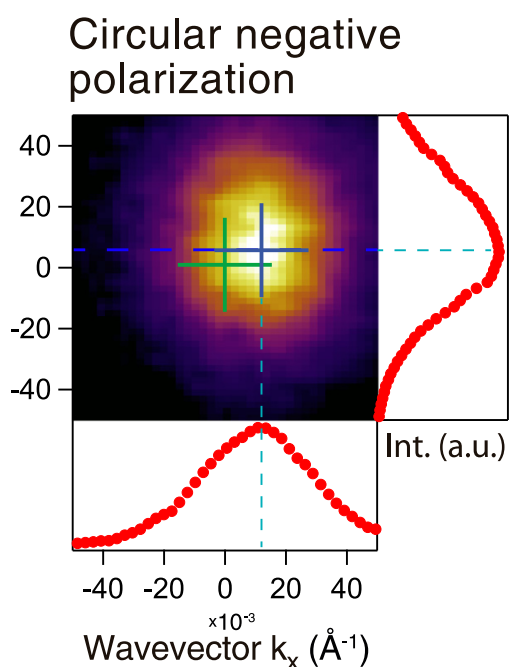

FIG. 5. (a) $\left(k_{x}, k_{y}\right)$ projections of the DC state maps cut at the energy near the Fermi level measured at $55 \mathrm{~K}$ for $\mathrm{Bi}_{1.37} \mathrm{~V}_{0.03} \mathrm{Sb}_{0.6} \mathrm{Te}_{2} \mathrm{Se}_{\mathrm{sith}}$ opposite circular polarization of SR showing an opposite TSS intensity asymmetry. (b) The $\left(k_{x}, k_{y}\right)$ shift of the DP position generated by the SR-induced in-plane magnetization using linearly $p$-polarized SR (marked by green crosses in all insets) and circularly polarized SR of opposite chirality (marked by blue crosses). The position $k_{\|}=0$ corresponds to the position of maximum of intensity of the MDC profile measured using linearly polarized SR. (c) Comparison between the $k_{\|}$position of the intensity maximum in the MDC profiles measured at the DC states near the Fermi level and at the DP using different polarization of SR.

(Fig. 6S) confirms the calculated temperature dependence of the DP $k_{\|}$shift shown in Fig. 4(d). Under the excitation by a circularly polarized SR of opposite chirality the $k_{\|}$shift of the DC position in the $k_{x}$ direction is larger, which is related to the higher TSS intensity asymmetry [see corresponding profiles, Fig. 5(c)]. However, this large shift of the DP is mainly related to the intensity redistribution and nonzero angular and energy acceptance of the electron analyzer; the real $k_{\|}$shift is indeed lower (see below).

For checking the $k_{\|}$shift of the DC position induced by the TSS intensity asymmetry two ways are possible, as follows.

(a) To create the TSS intensity asymmetry of opposite sign by circularly polarized SR with opposite chirality and to measure corresponding relative shift of the DC states and the $k_{\|}$position of the DC between these two cases at the same other conditions (see Figs. 5, 6, and Fig. 7S in the Supplemental Material [41]).

(b) To change the TSS intensity asymmetry by the photon energy variation (Figs. 1, 2, and 3, and Figs. $1 \mathrm{~S}$ and $2 \mathrm{~S}$ in the Supplemental Material [41], and see Figs. 7 and 8 below).

For testing the first possibility of the SR-induced $k_{\|}$shift in more detail, Fig. 6 demonstrates the MDC profiles for the TSS peaks measured in a separate experiment directly at the DP and at different cutting energies above the DP. Here, the $k_{\|}$shift between the MDC profiles measured at different cutting energies above the DP along the DC states for the cases of photoexcitation by circularly polarized SR of opposite chirality for $\mathrm{Bi}_{1.37} \mathrm{~V}_{0.03} \mathrm{Sb}_{0.6} \mathrm{Te}_{2} \mathrm{Se}$ is presented. For the experiment presented in Figs. 5(b) and 5(c) the $k_{\|}$ shift of the DC states at the DP of about $(22-24) \times 10^{-3} \AA^{-1}$ 

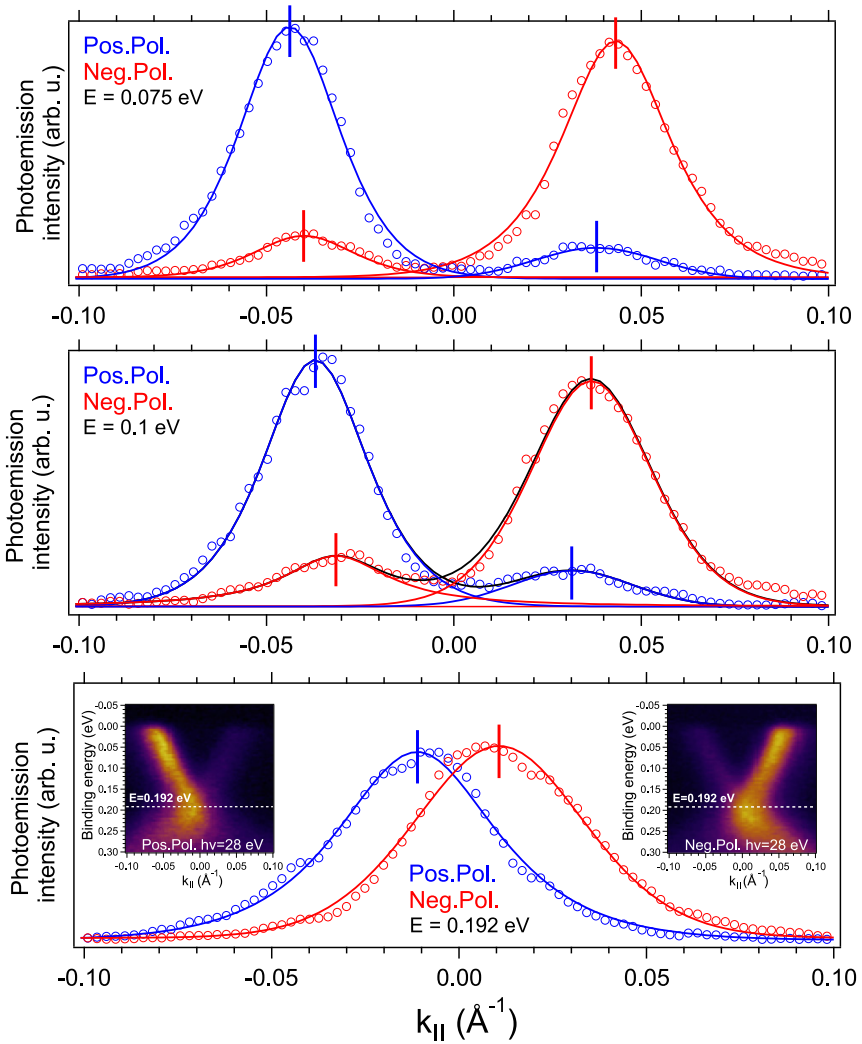

FIG. 6. Experimentally observed MDC profiles of the DC states and their $k_{\|}$shift measured under photoexcitation by circularly polarized SR of opposite chirality at the DP (bottom; insets show the ARPES maps) and at different cutting energies (middle and top) relative to the Fermi level above the DP for $\mathrm{Bi}_{1.37} \mathrm{~V}_{0.03} \mathrm{Sb}_{0.6} \mathrm{Te}_{2} \mathrm{Se}$.

can be observed. The experiment presented in Fig. 6 was carried out for the same sample after some time at different experimental conditions (swept mode with reduced slit size). In this case the $k_{\|}$shift of about $(21-22) \times 10^{-3} \AA^{-1}$ can be distinguished at the DP $(E=0.192 \mathrm{eV})$. However, at the cutting energies above the DP, where the opposite DC branches are separated $(E=0.15-0.075 \mathrm{eV})$, the $k_{\|}$shift between the cases of opposite circular polarizations is reduced down to the value of about $(4-5.5) \times 10^{-3} \AA^{-1}$. For cutting energies above the DP an approximately constant value of the $k_{\|}$shift is retained with a small decrease under a shift towards the Fermi level, while close to the Fermi level it is reduced to (3-3.5) $\times 10^{-3} \AA^{-1}$; see, for instance, Fig. 5(c). If we scan the energies along the upper DC the statistically averaged value is estimated to be about $4.5 \times 10^{-3} \AA^{-1} \pm 1.5 \times 10^{-3} \AA^{-1}$. Some distortion of the DC structure (as it was noted in Ref. [56]), especially near the Fermi level, can be related to the zero-bias spin-polarized current induced by the SR-generated uncompensated spin accumulation [8-10]. At the same time, a very large value of the $k_{\|}$shift observed at the DP is mainly related to an averaging over cutting energies above and below the DP with asymmetric redistribution of the TSS branch intensity at opposite circular polarizations of SR owing to the nonzero angular and energy acceptance; see the dispersions in the insets of Fig. 6 (bottom). Analogous tendencies for the $k_{\|}$ (a)
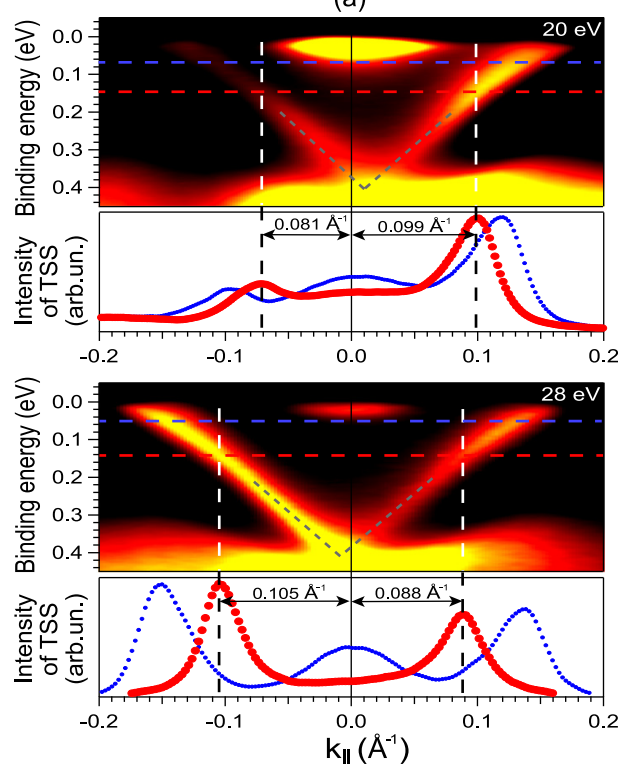

(b)
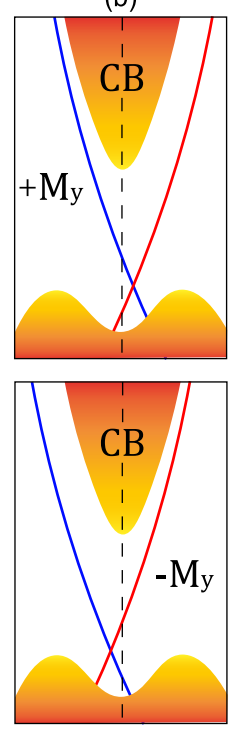

FIG. 7. (a) Experimental ARPES dispersion maps for $\mathrm{Bi}_{1.97} \mathrm{~V}_{0.03} \mathrm{Te}_{2.4} \mathrm{Se}_{0.6}$ measured at temperature $17 \mathrm{~K}$ in the $\overline{\Gamma M}$ direction at photon energies of 20 and $28 \mathrm{eV}$. The reversing of the asymmetry in the intensity of the DC states with opposite momentum at these photon energies is observed. Below each ARPES dispersion map the corresponding MDC profile of intensity of DC and bottom $\mathrm{CB}$ states measured at the cutting energies marked by the horizontal blue and red lines are presented. The cutting energies were chosen for showing the $\mathrm{CB}$ states close to the $\mathrm{CB}$ edge (blue lines) and well-defined intensity asymmetry for the DC states (red line). (b) Schematic presentation of the $k_{\|}$shift of the DC states relative to the non-spin-polarized $\mathrm{CB}$ states at different orientation of in-plane magnetic fields $\left(M_{y}\right)$.

shift with some distortions of the DC (in comparison with the rigid shift model) are observed for V-doped TIs of different stoichiometry, for instance, for $\mathrm{Bi}_{1.33} \mathrm{~V}_{0.03} \mathrm{Sb}_{0.66} \mathrm{Te}_{3}$, even if with different values of the $k_{\|}$shift. The corresponding ARPES dispersion maps and the MDC profiles under photoexcitation by SR of opposite chirality is presented in the Supplemental Material [41] (Fig. 7S). Therefore, one can conclude that indeed the $k_{\|}$shift of about $(4-7) \times 10^{-3} \AA^{-1}$ over the whole upper DC branches with some distortion of the DC structure can be distinguished experimentally for the V-doped TIs of different stoichiometry under photoexcitation by circularly polarized SR of opposite chirality.

The second way to confirm the in-plane magnetization induced by a linearly polarized SR can be connected with analysis of the $k_{\|}$shift of the spin-polarized DC states relative to the non-spin-polarized bottom CB states. A similar $k_{\|}$shift relative to the $\mathrm{CB}$ at the Fermi level under applied in-plane magnetic field was noted in Ref. [8]. Schematically, the $k_{\|}$ shift relative to the $\mathrm{CB}$ states depending on the direction of the in-plane magnetic field is shown in Fig. 7(b) (right column). Figures 7(a) and 8(a) demonstrate the experimental ARPES dispersion maps measured for $\mathrm{Bi}_{1.97} \mathrm{~V}_{0.03} \mathrm{Te}_{2.4} \mathrm{Se}_{0.6}$ and $\mathrm{Bi}_{2} \mathrm{Te}_{2} \mathrm{Se}$ showing the $k_{\|}$shift of the DC states relative to the bottom $\mathrm{CB}$ states located near the Fermi level. The dispersion maps were measured at different photon energies at 
(a)

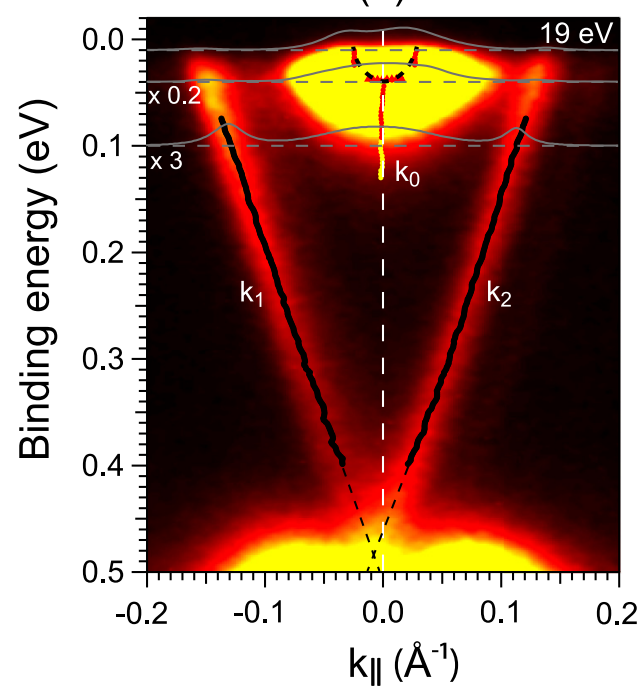

(b)

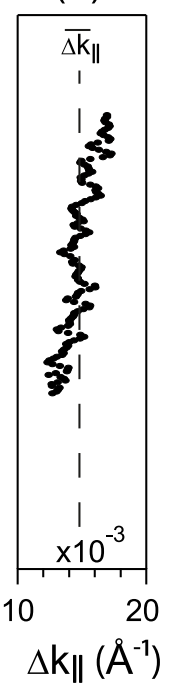

(c)

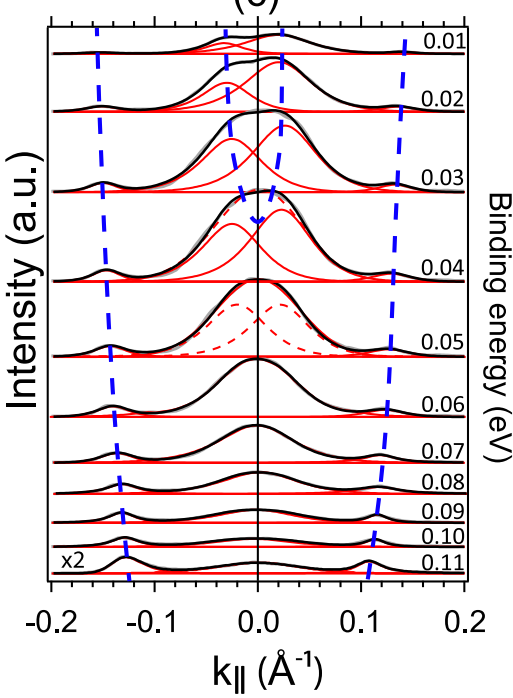

FIG. 8. (a) Experimental ARPES dispersion map for $\mathrm{Bi}_{2} \mathrm{Te}_{2} \mathrm{Se}$ measured at temperature $17 \mathrm{~K}$ in the $\overline{\Gamma K}$ direction at photon energy of $19 \mathrm{eV}$ showing the $k_{\|}$shift of the DC states relative to the bottom CB states connected with the asymmetry in the intensity of the DC states with opposite momentum. Here, approximations of the DC and CB states' positions obtained as a result of fitting procedure are shown by black and red lines, respectively, in comparison with MDC profiles in the region of the CB states. (b) Statistically estimated $k_{\|}$-shift variation measured along the DC branches at different cutting energies (with simultaneous fitting of both DC peaks in the MDC profiles). (c) Fitting of the MDC profiles for the $\mathrm{CB}$ states cut at different energies relative to the Fermi level with decomposition into spectral components. Here, the profiles of the DC states and their $k_{\|}$positions are presented, for comparison.

temperature of $17 \mathrm{~K}$ for $\mathrm{Bi}_{1.97} \mathrm{~V}_{0.03} \mathrm{Te}_{2.4} \mathrm{Se}_{0.6}$ [Fig. 7(a)] along $\overline{\Gamma M}$ (Geometry 1 ), when the TSS intensity asymmetry is the strongest, and for $\mathrm{Bi}_{2} \mathrm{Te}_{2} \mathrm{Se}$ (Fig. 8) in the $\overline{\Gamma K}$ direction $(\mathrm{Ge}-$ ometry 1). The ARPES maps demonstrate clearly a pronounced asymmetry in the intensity of the DC states with opposite momentum, which is inverted at the photon energies presented in Fig. 7 (similar to Fig. 1). In the bottom parts in Fig. 7 the corresponding MDC profiles of the intensity of the DC states with opposite momentum are presented in comparison with those for the bottom CB states arranged at the Fermi level. The cutting energies for the MDC profile measurements are marked by blue and red lines. The bottom CB states can be used to estimate the $\bar{\Gamma}$-point position (at $k_{\|}=0$ ).

Figures 7(a) and 8(a) demonstrate that the $k_{\|}$shift of the DC states relative to the bottom CB states is actually experimentally observed both for the $\mathrm{V}$-doped and the pristine TIs. The sign of the $k_{\|}$shift is inverted at the presented photon energies in relation to the change of the sign of the TSS intensity asymmetry with the photon energy. For $\mathrm{Bi}_{1.97} \mathrm{~V}_{0.03} \mathrm{Te}_{2.4} \mathrm{Se}_{0.6}$, the $k_{\|}$shift of 18 and $17 \times 10^{-3} \AA^{-1}$ (determined as a difference between the TSS peaks' positions for positive and negative $k_{\|}$) can be distinguished at $h v=20$ and $28 \mathrm{eV}$, respectively. This shift is developed in the opposite $k_{\|}$direction. With reducing the TSS intensity asymmetry for other photon energies the value of the $k_{\|}$shift tends to zero. These values correspond to the difference in the $k_{\|}$positions of the DC states with opposite momentum estimated relative to the bottom CB state position. In relation to it, the $k_{\|}$shift of the DP relative to $k_{\|}=0$ should be two times smaller than the $k_{\|}$shift between the opposite branches of the DC states. In Fig. 7 an extrapolation of the DC branches down to the intersection at the DP are presented by dashed lines. One can see that the $k_{\|}$shift of the DP relative to $k_{\|}=0$ (determined from the bottom CB states) in Fig. 7 is of about $9 \times 10^{-3} \AA^{-1}$, i.e., approximately twice smaller than the difference between the $k_{\|}$shifts of opposite DC branches noted above. Moreover, the sign of the $k_{\|}$shift is inverted in relation to the change of the sign in the TSS intensity asymmetry for $h v=20$ and $28 \mathrm{eV}$.

Figure 8 (b) shows the statistically estimated $k_{\|}$-shift variation measured for $\mathrm{Bi}_{2} \mathrm{Te}_{2}$ Se along the DC branches at different cutting energies (with simultaneous fitting of both DC peaks in the MDC profiles). It demonstrates a small decrease of the $k_{\|}$shift from $\sim(17-18) \times 10^{-3} \AA^{-1}$ to $\sim 12 \times 10^{-3} \AA^{-1}$ with a shift towards the DP. Closer to the DP the tendency to decrease the $k_{\|}$shift slows down to an approximately constant level. The $k_{\|}$shift at the DP relative to the bottom DC states $\left(k_{\|}=0\right)$ can be estimated on the level of about $6 \times 10^{-3} \AA^{-1}$ (twice smaller). The possibility of some distortion of the DC structure under influence of the induced magnetic field was noted in Ref. [55]. As one can see from Fig. 8(b), the mean-square deviation in the $k_{\|}$-shift value can be estimated on the level of about $2 \times 10^{-3} \AA^{-1}$. The accuracy of the $k_{\|}$-shift estimation relative to the CB states (as an indicator of the $\bar{\Gamma}$-point position) depends also on the asymmetry redistribution of the CB state connected, for instance, with the final state effects. Additionally, for TIs, the bottom CB states measured by ARPES are often described by parabolic Rashba split states with possible redistribution of the intensity with photon energy. Figure 8(c) shows the fitting of the MDC profiles in the region of the $\mathrm{CB}$ state localization at a series of cutting energies relative to the Fermi level. Closer to the Fermi level the fitting shows a decomposition into two spectral components with varying intensities depending on 

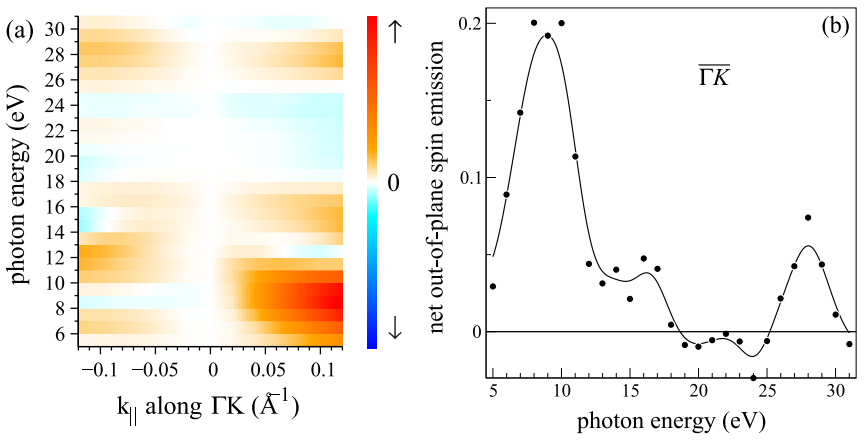

FIG. 9. (a) Calculated photon-energy dependence of the $k_{\|}$distribution $S\left(k_{\|}, h v\right)$ of the net out-of-plane spin in photoelectrons excited by a $p$-polarized SR along the SR incidence plane $(\overline{\Gamma K})$ from the upper DC of $\mathrm{Bi}_{2} \mathrm{Te}_{2} \mathrm{Se}$ for $h v$ from 5 to $31 \mathrm{eV}$. (b) Photon energy dependence of the net out-of-plane spin integrated over $k_{\|}$from -0.12 to $0.12 \AA^{-1}$ along $\overline{\Gamma K}$.

the cutting energy. However, despite the redistribution of the spectral component intensity near the Fermi level, at the bottom of the CB states the overall $\mathrm{CB}$ state distribution demonstrates rather one broadened peak. We ascribe the $k_{\|}$position of the peak at the bottom of the CB states to the $\bar{\Gamma}$ point with $k_{\|}=0$. The fitting of the CB spectrum at the Fermi level by two peaks shows that these peaks are also symmetrized relative to the position $k_{\|}=0$ estimated by using the bottom $\mathrm{CB}$ states. The analysis of the inaccuracy in the $k_{\|}$shift measured relative to the bottom of the $\mathrm{CB}$ states is presented in the Supplemental Material [41]. The overall $k_{\|}$-shift inaccuracy can be estimated on the level of about $(3-4) \times 10^{-3} \AA^{-1}$ (for the spectra with normal statistics). It is smaller than the value of the experimentally observed $k_{\|}$shifts.

So, we can conclude that the MDC profiles clearly demonstrate the $k_{\|}$shift of the TSS branches relative to the bottom CB states. It is important that the direction of the $k_{\|}$shift is related to the observed TSS intensity asymmetry. With inversion of the TSS intensity asymmetry at different photon energy the $k_{\|}$ shift of the TSS branches relative to the bottom CB states is also inverted. This opposite $k_{\|}$shift of the DC states testifies to the induced in-plane magnetic field orientation depending on the direction of the TSS intensity asymmetry.

Thus the variation of the TSS intensity asymmetry with photon energy or with helicity of the circular polarized light is followed by a $k_{\|}$shift of the DC states relative to the $\bar{\Gamma}$ point in accordance with the sign and the value of the observed TSS intensity asymmetry. This finding confirms that the observed $k_{\|}$shift is indeed connected with the SR-induced in-plane magnetization. The observation of the $k_{\|}$shift of the DP position can be a good indicator of the induced in-plane magnetic field due to the nonequal depopulation of the TSSs with opposite momenta.

\section{Possibility of out-of-plane induced magnetization}

Let us now discuss the problem of the out-of-plane magnetization induced by a linearly polarized SR. Figure 9(a) shows the calculated photon energy dependence of the out-of-plane net-spin photocurrent $S\left(k_{\|}, h v\right)=I^{\uparrow}\left(k_{\|}, h v\right)-$
$I^{\downarrow}\left(k_{\|}, h v\right)$ from the upper DC of $\mathrm{Bi}_{2} \mathrm{Te}_{2}$ Se with the SR incident along $\overline{\Gamma K}$. The out-of-plane net-spin photocurrent integrated over $k_{\|}$does not vanish, and its photon energy dependence is shown in Fig. 9(b). The magnitude and the sign of the integrated photocurrent are seen to vary with the photon energy, which suggests that also the total out-of-plane spin accumulation may be different for different photon energies. This out-of-plane spin accumulation induces an out-of-plane magnetization, which lifts the degeneracy of the TSSs and opens an energy gap at the DP due to the TRS breaking, as in Refs. [14,57] under a circularly polarized SR. Using Eq. (7) one can estimate the energy gap as $\Delta=2 \frac{\tilde{a}^{2}}{\mu_{B}} J_{e V} m_{z}^{V}+2 \frac{\tilde{a}^{2}}{\mu_{B}} U m_{z}$.

Figure 4(c) shows the calculated temperature dependence of the gap induced at the Dirac point using the value of the TSS asymmetry of 0.3 (averaged over the asymmetry values presented in Fig. 2 at $h v=28 \mathrm{eV}$ ) at the experimental conditions noted above (see Methods section for details). For magnetically doped TI with $3 \%$ of the V doping level below $30 \mathrm{~K}$ a gap of about $25 \mathrm{meV}$ is predicted. As the temperature increases, the gap decreases down to $12-15 \mathrm{meV}$ at room temperature. A finite gap above the Curie temperature is just related to the out-of-plane magnetization generated by SR. In the case of pristine TI a formation of the gap of $4.5 \mathrm{meV}$ is expected too, independent of temperature. However, our energy resolution was not high enough to allow the measurement of this small gap value for the pristine TI.

The SR-induced out-of-plane surface magnetization can be experimentally tested by the splitting of the DC states in the ARPES dispersions and opening of the energy gap at the DP. In accordance with the theoretical estimations [Fig. 4(c)] the value of the gap at the DP at low temperature for 3\% V-doped TI is expected to be of about $25 \mathrm{meV}$. Figure $9 \mathrm{~S}$ in the Supplemental Material [41] shows the ARPES dispersion maps in the form of $N(E)$ and $d^{2} N / d E^{2}$ presentation and corresponding EDC at the DP measured at the $1^{3}$ end station at BESSY II (HelmholtzZentrum Berlin) for $\mathrm{V}$-doped TIs $\mathrm{Bi}_{1.31} \mathrm{~V}_{0.03} \mathrm{Sb}_{0.66} \mathrm{Te}_{3}$ and $\mathrm{Bi}_{1.3} \mathrm{~V}_{0.04} \mathrm{Sb}_{0.66} \mathrm{Te}_{3}$ at the temperatures of $1 \mathrm{~K}$ and $66 \mathrm{~K}$. These data assume the splitting between the DC states at the DP of about 25 and $20 \mathrm{meV}$, respectively, which correlate with the gap values predicted in Fig. 4. Unfortunately, due to the error introduced by finite analyzer slit size and the corresponding angle- and energy-spectral averaging the data presented in the Supplemental Material [41] (Fig. 9S) cannot give direct and correct information about the gap formation of the values noted above. See the Supplemental Material [41] (Fig. 8S) for the analysis of the introduced error. Therefore, in order to test experimentally an influence of the induced out-of-plane magnetization we present in Fig. 10 the corresponding ARPES dispersion maps and EDC profiles measured directly at the DP for V-doped TIs with stoichiometry $\mathrm{Bi}_{1.09} \mathrm{~V}_{0.06} \mathrm{Sb}_{0.85} \mathrm{Te}_{3}$, with twice higher $\mathrm{V}$ concentration. These spectra were measured at the $1^{2}$ end station at BESSY II (Helmholtz-Zentrum Berlin) at temperature $55 \mathrm{~K}$. The measured ARPES dispersion maps are presented in the forms of $N(E)$ and $d^{2} N / d E^{2}$ [lines (a) and (b), respectively] for better visualization of the DC state splitting at the DP. For testing the influence of SR on the induced out-of-plane magnetization the dispersion maps measured with using different polarizations of SR are presented for comparison. 

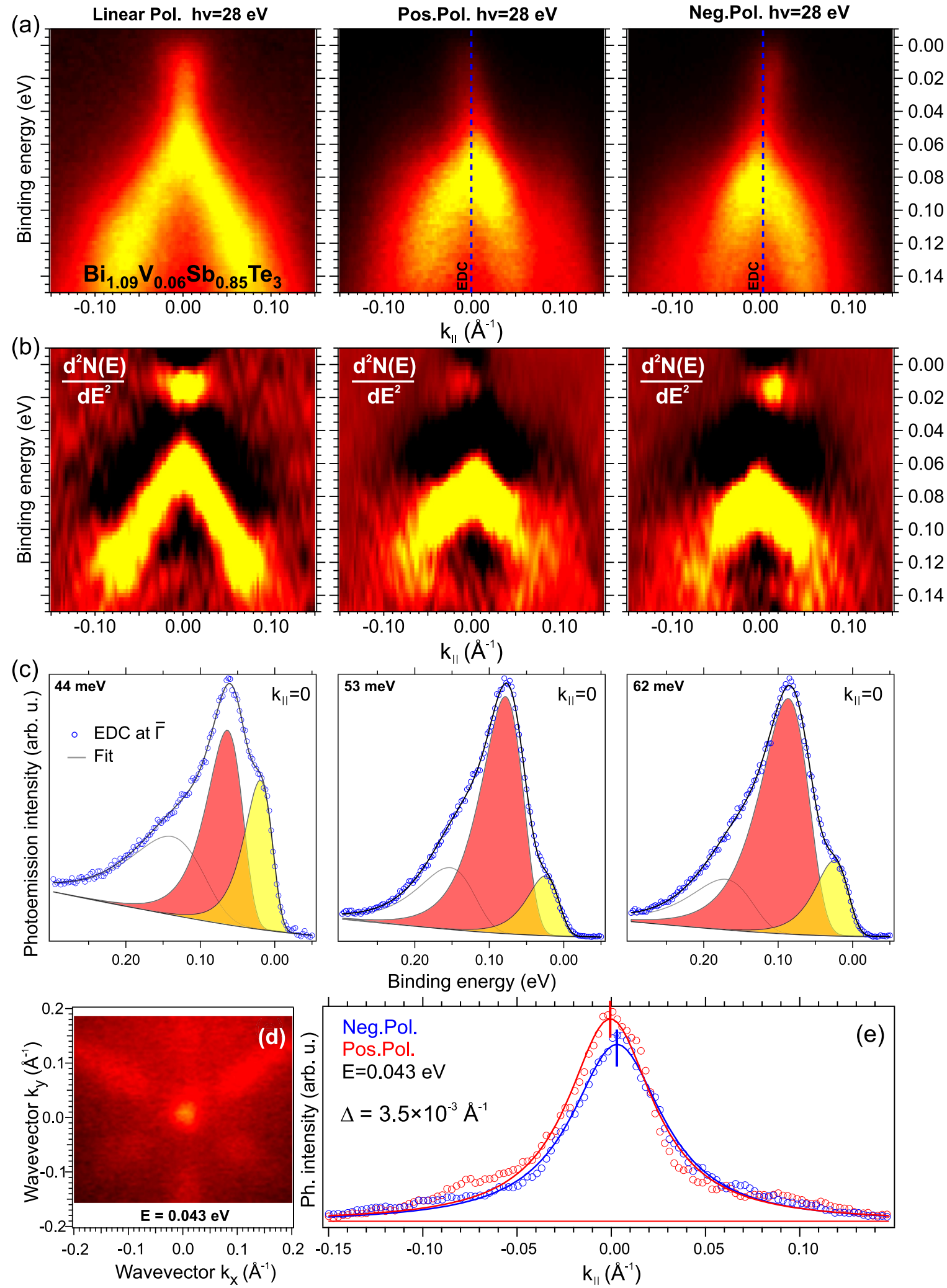

FIG. 10. (a) ARPES intensity maps measured for V-doped TIs with stoichiometry $\mathrm{Bi}_{1.09} \mathrm{~V}_{0.06} \mathrm{Sb}_{0.85} \mathrm{Te}_{3}$ under photoexcitation by SR with different polarizations at temperature of $55 \mathrm{~K}$. The corresponding dispersions in the $d^{2} N / d E^{2}$ form are shown in the middle line (b) for better visualization of the DC states splitting at the DP. Line (c): corresponding EDCs measured at the DP $\left(k_{\|}=0\right)$ and the result of the fitting procedure with decomposition into spectral components. (d) The $k_{x}, k_{y}$ mapping at the DP $(E=0.043 \mathrm{eV})$ and (e) the $k_{\|}$shift of the DC states at the DP developed under photoexcitation by SR of opposite chirality. 


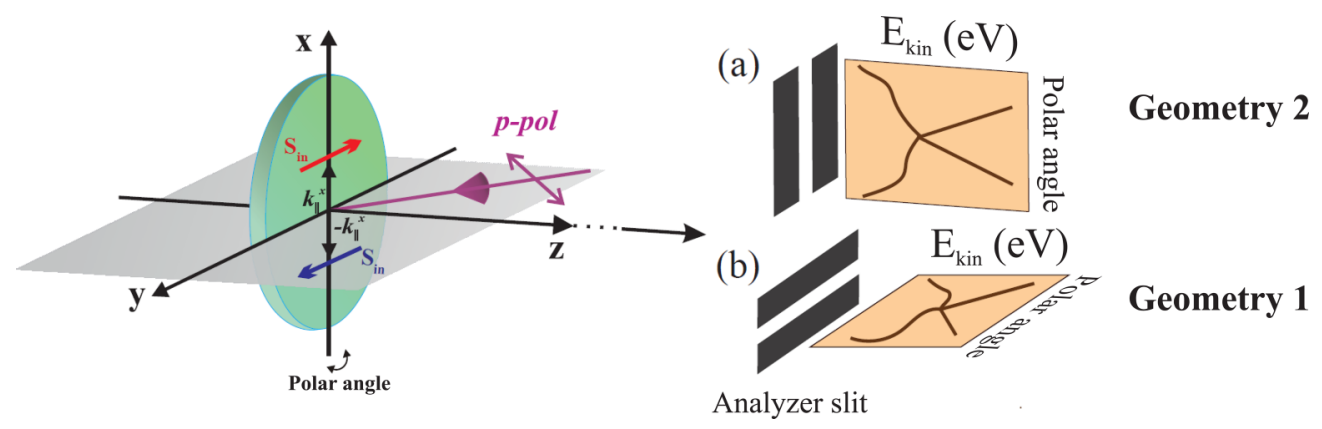

FIG. 11. METHODS section. Schematic presentation of the ARPES measurement geometries with the analyzer slit oriented along (Geometry 1) and perpendicular to (Geometry 2) the SR incidence plane.

This kind of V-doped TI is characterized by arrangement of the DP and corresponding Dirac gap close to the Fermi level that is very important for effective realization of the QAHE [18-20]. The presented ARPES dispersion map in the $d^{2} N / d E^{2}$ form and corresponding EDC [in line (c)] measured with linearly polarized SR demonstrate the splitting of the DC states at the DP. Figure 10(d) shows the the $k_{x}, k_{y}$ mapping at the DP, which confirms that the data presented in Fig. 10, lines (a)-(c) were measured at the DP at $k_{\|}=0$. The fitting of the EDC profiles at the DP shows decomposition into two spectral components (red and yellow peaks) close to the DP with an energy splitting of about $44 \mathrm{meV}$. It is approximately twice higher than that for the V-doped TI with the $\mathrm{V}$ concentration of 3\% (Fig. 4 and Fig. 9S in the Supplemental Material [41]) and can testify to the splitting of the DC states at the DP despite the influence of the error introduced by the finite analyzer slit size noted above (see Fig. 8S in the Supplemental Material [41] for details). The upper DC states are practically unoccupied. Only the lower part near the Dirac gap located at the Fermi level is visible in the ARPES dispersion maps. Under photoexcitation by circularly polarized SR of opposite chirality the corresponding reversal redistribution in intensity of the upper DC states (and in some degree of the lower DC) is observed. It is very important that the splitting of the DC states at the DP under photoexcitation by circularly polarized SR increases up to 53 and $62 \mathrm{meV}$ [see Fig. 10, line (c)]. If we assume that the maximum of intensity of the upper DC states can be located above the Fermi level then the estimated value of the DC state splitting can be increased. In the bottom [Fig. 10(e)], the corresponding $k_{\|}$shift of the DC states at the DP is presented which is of about $3.5 \times 10^{-3} \AA^{-1}$. It means that for this kind of V-doped TI the out-of-plane component is mainly excited with reduced in-plane component. Moreover, the influence of SR (leading to the induced out-of-plane magnetization) can modulate (increase) the value of DC state splitting at the DP. This finding can be useful for the realization of QAHE modulating the conditions for realizing it and its increase to higher temperatures.

In summary, we have demonstrated that the intensity asymmetry of the DC states with opposite momentum generated by $p$-polarized SR is accompanied in magnetically doped and pristine TIs by a hole-generated uncompensated spin accumulation, which may give rise to an in-plane and outof-plane magnetic field. Experimentally, it is indicated by the $k_{\|}$shift of the DC position under the induced in-plane magnetic field and by the splitting of the TSSs at the DP induced by the out-of-plane component of magnetization even above the Curie temperature. Theoretical estimations have confirmed a possibility of the induced in-plane and out-ofplane magnetization by linearly polarized SR.

This finding should be taken into account in PE investigations of systems with helical spin structure, especially for magnetically doped TIs, where the photoionization by SR of different polarization and photon energy can influence the electronic and spin structure and induce the in-plane and out-of-plane magnetization in dependence on experimental conditions and stoichiometry of the TIs. Moreover, it can be used for more effective modulation and control of the conditions for the effective realization of the QAHE.

\section{METHODS}

The measurements of ARPES intensity maps for the DC states in pristine and magnetically doped TIs presented in Fig. 1 and in the Supplemental Material [41] (Figs. 1S and 2S) were carried out at i3 beamline at MAXlab (Lund, Sweden) and $\mathrm{BaDElPh}$ beamline at Elettra (Trieste, Italy) in the direction along the SR incidence plane (Geometry 1 in Fig. 11) and the $1^{2}$ (ARPES One-Square) end station at BESSY II (HelmholtzZentrum Berlin, Germany) in the direction perpendicular to the SR incidence plane (Geometry 2 in Fig. 11) using a VG Scienta R4000 or SPECS Phoibos 150 hemispherical energy analyzers. The incidence angle of SR for these experiments was $73^{\circ}$ (MAXlab) and $50^{\circ}$ (Elettra and BESSY II) relative to the surface normal.

The DC energy cut maps of the TSSs for $\mathrm{Bi}_{1.37} \mathrm{~V}_{0.03} \mathrm{Sb}_{0.6} \mathrm{Te}_{2} \mathrm{Se}$ (Fig. 5, 6 and Fig. $6 \mathrm{~S}$ in the Supplemental Material [41]) were measured at the $1^{2}$ (ARPES One-Square) end station at BESSY II (Helmholtz-Zentrum Berlin, Germany) with a photon energy of $28 \mathrm{eV}$ keeping the sample at the temperature of $55 \mathrm{~K}$ and room temperature, respectively. The $k_{\|}$shift of the DC states at different cutting energies measured for V-doped TI with different stoichiometry $\mathrm{Bi}_{1.31} \mathrm{~V}_{0.03} \mathrm{Sb}_{0.66} \mathrm{Te}_{3}$ (Fig. 7S in the Supplemental Material [41]) was measured at the $1^{3}$ station at BESSY II (Helmholtz-Zentrum Berlin, Germany) at a temperature of $1 \mathrm{~K}$.

The ARPES dispersion maps for magnetically doped TIs (Fig. 10 and Fig. 9S in the Supplemental Material [41]), which were used for a careful estimation of the energy splitting of the TSSs at the DP, were measured at the $1^{2}$ (ARPES One- 
Square) and $1^{3}$ (ARPES One-Cube) end stations with enhanced energy and angle resolutions at BESSY II (Helmholtz-Zentrum Berlin, Germany) at the SR incidence angle of $45^{\circ}$ relative to the surface normal.

The ARPES dispersion maps for the estimation of the $k_{\|}$ shift relative to the non-spin-polarized bottom CB states at the Fermi level (Fig. 7 and Fig. 8) were measured at BaDElPh beamline at Elettra (Trieste, Italy).

Part of this work was carried out in the resource center "Physical methods of surface investigation" (PMSI) of Research park of Saint Petersburg State University.

The single crystals of pristine TIs $\mathrm{Bi}_{1.5} \mathrm{Sb}_{0.5} \mathrm{Te}_{1.8} \mathrm{Se}_{1.2}$, $\mathrm{Bi}_{2} \mathrm{Te}_{2} \mathrm{Se}$, and $\mathrm{Bi}_{1.4} \mathrm{Sb}_{0.6} \mathrm{Te}_{2} \mathrm{Se}$, and magnetically doped TIs $\mathrm{Bi}_{1.37} \mathrm{~V}_{0.03} \mathrm{Sb}_{0.6} \mathrm{Te}_{2} \mathrm{Se}, \mathrm{Bi}_{1.3} \mathrm{~V}_{0.04} \mathrm{Sb}_{0.66} \mathrm{Te}_{2} \mathrm{Se}$, $\mathrm{Bi}_{1.97} \mathrm{~V}_{0.03} \mathrm{Te}_{2.4} \mathrm{Se}_{0.6}$, and $\mathrm{Bi}_{1.09} \mathrm{~V}_{0.06} \mathrm{Sb}_{0.85} \mathrm{Te}_{3}$ were synthesized in Novosibirsk State University by using a modified vertical Bridgman method [58]. Clean surfaces of the TIs were obtained by a cleavage in ultrahigh vacuum.

The base pressure during the experiments was better than $1 \times 10^{-10}$ mbar.

\section{ACKNOWLEDGMENTS}

The authors acknowledge support by Saint Petersburg State University (Grant No. 15.61.202.2015), Russian Science Foundation Grant No. 17-12-01333 (in the part of theoretical study of magnetic properties), Russian Science Foundation Grant No. 18-12-00062 (in the part of ARPES measurements and analysis of the electronic structure modification under influence of SR), and Russian Science Foundation Grant No. 17-12-01047 (in part of crystal growth and the sample characterization). The work was also supported by the Spanish Ministry of Economy and Competitiveness MINECO (Project No. FIS2016-76617-P), German-Russian Interdisciplinary Science Center (G-RISC) funded by the German Federal Foreign Office via the German Academic Exchange Service (DAAD), and Russian-German laboratory at BESSY II (Helmholtz-Zentrum Berlin). The authors kindly acknowledge the MAXLab, HiSOR, Elettra, BESSY II, and RC PMSI of Saint Petersburg State University staff for technical support and help with experiment and useful discussions.
[1] M. Z. Hasan and C. L. Kane, Rev. Mod. Phys. 82, 3045 (2010).

[2] D. Hsieh, Y. Xia, D. Qian, L. Wray, J. H. Dil, F. Meier, J. Osterwalder, L. Patthey, J. G. Checkelsky, N. P. Ong, A. V. Fedorov, H. Lin, A. Bansil, D. Grauer, Y. S. Hor, R. J. Cava, and M. Z. Hasan, Nature (London) 460, 1101 (2009).

[3] H. Zhang, C.-X. Liu, X.-L. Qi, X. Dai, Z. Fang, and S.-C. Zhang, Nat. Phys. 5, 438 (2009).

[4] J. Moore, Nat. Phys. 5, 378 (2009).

[5] P. Hosur, Phys. Rev. B 83, 035309 (2011).

[6] A. Junck, G. Refael, and F. von Oppen, Phys. Rev. B 88, 075144 (2013).

[7] J. W. McIver, D. Hsieh, H. Steinberg, P. Jarillo-Herrero, and N. Gedik, Nat. Nano 7, 96 (2012).

[8] N. Ogawa, R. Yoshimi, K. Yasuda, A. Tsukazaki, M. Kawasaki, and Y. Tokura, Nat. Commun. 7, 12246 (2016).

[9] A. M. Shikin, A. A. Rybkina, I. I. Klimovskikh, M. V. Filyanina, K. A. Kokh, O. E. Tereshchenko, P. Skirdkov, K. Zvezdin, and A. Zvezdin, Phys. Solid State 58, 1675 (2016).

[10] C. Kastl, C. Karnetzky, H. Karl, and A. W. Holleitner, Nat. Commun. 6, 6617 (2015).

[11] C. H. Li, O. M. J. van‘t Erve, J. T. Robinson, Y. Liu, L. Li, and B. T. Jonker, Nat. Nano 9, 218 (2014).

[12] A. R. Mellnik, J. S. Lee, A. Richardella, J. L. Grab, P. J. Mintun, M. H. Fischer, A. Vaezi, A. Manchon, E.-A. Kim, N. Samarth, and D. C. Ralph, Nature (London) 511, 449 (2014).

[13] Y. Fan, P. Upadhyaya, X. Kou, M. Lang, S. Takei, Z. Wang, J. Tang, L. He, L.-T. Chang, M. Montazeri, G. Yu, W. Jiang, T. Nie, R. N. Schwartz, Y. Tserkovnyak, and K. L. Wang, Nat. Mater. 13, 699 (2014).

[14] A. M. Shikin, A. A. Rybkina, I. I. Klimovskikh, M. V. Filianina, K. A. Kokh, O. E. Tereshchenko, P. N. Skirdkov, K. A. Zvezdin, and A. K. Zvezdin, Appl. Phys. Lett. 109, 222404 (2016).

[15] X.-L. Qi, T. L. Hughes, and S.-C. Zhang, Phys. Rev. B 78, 195424 (2008).

[16] K. Nomura and N. Nagaosa, Phys. Rev. Lett. 106, 166802 (2011).

[17] J. Wang, B. Lian, X.-L. Qi, and S.-C. Zhang, Phys. Rev. B 92, 081107 (2015).
[18] C.-Z. Chang, J. Zhang, X. Feng, J. Shen, Z. Zhang, M. Guo, K. Li, Y. Ou, P. Wei, L.-L. Wang, Z.-Q. Ji, Y. Feng, S. Ji, X. Chen, J. Jia, X. Dai, Z. Fang, S.-C. Zhang, K. He, Y. Wang, L. Lu, X.-C. Ma, and Q.-K. Xue, Science 340, 167 (2013).

[19] C.-Z. Chang, W. Zhao, D. Y. Kim, H. Zhang, B. A. Assaf, D. Heiman, S.-C. Zhang, C. Liu, M. H. W. Chan, and J. S. Moodera, Nat. Mater. 14, 473 (2015).

[20] R. Yu, W. Zhang, H.-J. Zhang, S.-C. Zhang, X. Dai, and Z. Fang, Science 329, 61 (2010).

[21] M. Neupane, S.-Y. Xu, Y. Ishida, S. Jia, B. M. Fregoso, C. Liu, I. Belopolski, G. Bian, N. Alidoust, T. Durakiewicz, V. Galitski, S. Shin, R. J. Cava, and M. Z. Hasan, Phys. Rev. Lett. 115, 116801 (2015).

[22] A. Crepaldi, F. Cilento, B. Ressel, C. Cacho, J. C. Johannsen, M. Zacchigna, H. Berger, P. Bugnon, C. Grazioli, I. C. E. Turcu, E. Springate, K. Kern, M. Grioni, and F. Parmigiani, Phys. Rev. B 88, 121404 (2013).

[23] M. Hajlaoui, E. Papalazarou, J. Mauchain, L. Perfetti, A. TalebIbrahimi, F. Navarin, M. Monteverde, P. Auban-Senzier, C. Pasquier, N. Moisan, D. Boschetto, M. Neupane, M. Hasan, T. Durakiewicz, Z. Jiang, Y. Xu, I. Miotkowski, Y. Chen, S. Jia, H. Ji, R. Cava, and M. Marsi, Nat. Commun. 5, 3003 (2014).

[24] A. Chernyshov, M. Overby, X. Liu, J. K. Furdyna, Y. LyandaGeller, and L. P. Rokhinson, Nat. Phys. 5, 656 (2009).

[25] H. Zhang, C.-X. Liu, and S.-C. Zhang, Phys. Rev. Lett. 111, 066801 (2013).

[26] Y. Cao, J. A. Waugh, X.-W. Zhang, J.-W. Luo, Q. Wang, T. J. Reber, S. K. Mo, Z. Xu, A. Yang, J. Schneeloch, G. D. Gu, M. Brahlek, N. Bansal, S. Oh, A. Zunger, and D. S. Dessau, Nat. Phys. 9, 499 (2013).

[27] Z.-H. Zhu, C. N. Veenstra, G. Levy, A. Ubaldini, P. Syers, N. P. Butch, J. Paglione, M. W. Haverkort, I. S. Elfimov, and A. Damascelli, Phys. Rev. Lett. 110, 216401 (2013).

[28] Z.-H. Zhu, C. N. Veenstra, S. Zhdanovich, M. P. Schneider, T. Okuda, K. Miyamoto, S.-Y. Zhu, H. Namatame, M. Taniguchi, M. W. Haverkort, I. S. Elfimov, and A. Damascelli, Phys. Rev. Lett. 112, 076802 (2014). 
[29] C. Seibel, J. Braun, H. Maaß, H. Bentmann, J. Minár, T. V. Kuznetsova, K. A. Kokh, O. E. Tereshchenko, T. Okuda, H. Ebert, and F. Reinert, Phys. Rev. B 93, 245150 (2016).

[30] C. Jozwiak, C.-H. Park, K. Gotlieb, C. Hwang, D.-H. Lee, S. G. Louie, J. D. Denlinger, C. R. Rotundu, R. J. Birgeneau, Z. Hussain, and A. Lanzara, Nat. Phys. 9, 293 (2013).

[31] C.-H. Park and S. G. Louie, Phys. Rev. Lett. 109, 097601 (2012).

[32] C. Jozwiak, Y. L. Chen, A. V. Fedorov, J. G. Analytis, C. R. Rotundu, A. K. Schmid, J. D. Denlinger, Y.-D. Chuang, D.-H. Lee, I. R. Fisher, R. J. Birgeneau, Z.-X. Shen, Z. Hussain, and A. Lanzara, Phys. Rev. B 84, 165113 (2011).

[33] M. Neupane, S. Basak, N. Alidoust, S.-Y. Xu, C. Liu, I. Belopolski, G. Bian, J. Xiong, H. Ji, S. Jia, S.-K. Mo, M. Bissen, M. Severson, H. Lin, N. P. Ong, T. Durakiewicz, R. J. Cava, A. Bansil, and M. Z. Hasan, Phys. Rev. B 88, 165129 (2013).

[34] S.-Y. Xu, M. Neupane, C. Liu, D. Zhang, A. Richardella, L. Andrew Wray, N. Alidoust, M. Leandersson, T. Balasubramanian, J. Sanchez-Barriga, O. Rader, G. Landolt, B. Slomski, J. Hugo Dil, J. Osterwalder, T.-R. Chang, H.-T. Jeng, H. Lin, A. Bansil, N. Samarth, and M. Zahid Hasan, Nat. Phys. 8, 616 (2012).

[35] G. Rosenberg and M. Franz, Phys. Rev. B 85, 195119 (2012).

[36] T. Hirahara, S. V. Eremeev, T. Shirasawa, Y. Okuyama, T. Kubo, R. Nakanishi, R. Akiyama, A. Takayama, T. Hajiri, S.-i. Ideta, M. Matsunami, K. Sumida, K. Miyamoto, Y. Takagi, K. Tanaka, T. Okuda, T. Yokoyama, S.-i. Kimura, S. Hasegawa, and E. V. Chulkov, Nano Lett. 17, 3493 (2017).

[37] M. M. Otrokov, T. V. Menshchikova, M. G. Vergniory, I. P. Rusinov, A. Y. Vyazovskaya, Y. M. Koroteev, G. Bihlmayer, A. Ernst, P. M. Echenique, A. Arnau, and E. V. Chulkov, 2D Mater. 4, 025082 (2017).

[38] K. Miyamoto, A. Kimura, T. Okuda, H. Miyahara, K. Kuroda, H. Namatame, M. Taniguchi, S. V. Eremeev, T. V. Menshchikova, E. V. Chulkov, K. A. Kokh, and O. E. Tereshchenko, Phys. Rev. Lett. 109, 166802 (2012).

[39] A. M. Shikin, I. I. Klimovskikh, S. V. Eremeev, A. A. Rybkina, M. V. Rusinova, A. G. Rybkin, E. V. Zhizhin, J. Sánchez-Barriga, A. Varykhalov, I. P. Rusinov, E. V. Chulkov, K. A. Kokh, V. A. Golyashov, V. Kamyshlov, and O. E. Tereshchenko, Phys. Rev. B 89, 125416 (2014).
[40] C. S. Tang, B. Xia, X. Zou, S. Chen, H.-W. Ou, L. Wang, A. Rusydi, J.-X. Zhu, and E. E. M. Chia, Sci. Rep. 3, 3513 (2013).

[41] See Supplemental Material at http://link.aps.org/supplemental/ 10.1103/PhysRevB.97.245407 for additional ARPES data analysis, analysis of the inaccuracy in the $k_{\|}$-shift determination, and details of theoretical consideration.

[42] I. Adawi, Phys. Rev. 134, A788 (1964).

[43] E. E. Krasovskii and W. Schattke, Phys. Rev. B 59, R15609 (1999).

[44] E. E. Krasovskii, F. Starrost, and W. Schattke, Phys. Rev. B 59, 10504 (1999).

[45] D. D. Koelling and B. N. Harmon, J. Phys. C 10, 3107 (1977).

[46] A. M. Shikin, V. Y. Voroshin, A. G. Rybkin, K. A. Kokh, O. E. Tereshchenko, Y. Ishida, and A. Kimura, 2D Mater. 5, 015015 (2018).

[47] Physical Properties of Crystals: Their Representation by Tensors and Matrices, edited by J. F. Nye (Clarendon Press, London, 1985).

[48] Symmetry and Magnetism, edited by R. Birss (Holland Publ. Co., Amsterdam, 1964).

[49] Y. I. Sirotin and M. P. Shaskolskaya, Basic Crystallophysics (Science, Moscow, 1979).

[50] F. J. DiSalvo, Science 285, 703 (1999).

[51] Y. Xia, D. Qian, D. Hsieh, L. Wray, A. Pal, H. Lin, A. Bansil, D. Grauer, Y. S. Hor, R. J. Cava et al., Nat. Phys. 5, 398 (2009).

[52] Optical Orientation, edited by F. Meier and B. P. Zakharchenya (Elsevier, Amsterdam, 2012).

[53] Q. Liu, C.-X. Liu, C. Xu, X.-L. Qi, and S.-C. Zhang, Phys. Rev. Lett. 102, 156603 (2009).

[54] J.-J. Zhu, D.-X. Yao, S.-C. Zhang, and K. Chang, Phys. Rev. Lett. 106, 097201 (2011).

[55] J. Henk, M. Flieger, I. V. Maznichenko, I. Mertig, A. Ernst, S. V. Eremeev, and E. V. Chulkov, Phys. Rev. Lett. 109, 076801 (2012).

[56] Y. G. Semenov, X. Li, and K. W. Kim, Phys. Rev. B 86, 201401 (2012).

[57] A. M. Shikin, A. A. Rybkina, I. I. Klimovskikh, O. E. Tereshchenko, A. S. Bogomyakov, K. A. Kokh, A. Kimura, P. N. Skirdkov, K. A. Zvezdin, and A. K. Zvezdin, 2D Mater. 4, 025055 (2017).

[58] K. A. Kokh, S. V. Makarenko, V. A. Golyashov, O. A. Shegai, and O. E. Tereshchenko, CrystEngComm 16, 581 (2014). 